\title{
Activation-Dependent Rapid Postsynaptic Clustering of Glycine Receptors in Mature Spinal Cord Neurons
}

\author{
(1) Yoshihisa Nakahata, ${ }^{1,2}$ Kei Eto, ${ }^{1,2}$ Hideji Murakoshi, ${ }^{2,3,4}$ Miho Watanabe, ${ }^{5}$ (D) Toshihiko Kuriu, ${ }^{6}$ Hiromi \\ Hirata ${ }^{4,7,8,9}$, Andrew J. Moorhouse, ${ }^{10}$ Hitoshi Ishibashi, ${ }^{1,2,11}$ and Junichi Nabekura ${ }^{1,2,12}$
}

DOI:http://dx.doi.org/10.1523/ENEURO.0194-16.2017

\begin{abstract}
${ }^{1}$ Division of Homeostatic Development, Department of Developmental Physiology, National Institute for Physiological Sciences, Okazaki 444-8585, Japan, ${ }^{2}$ Department of Physiological Sciences, The Graduate University for Advanced Studies (SOKENDAI), Okazaki 444-8585, Japan, ${ }^{3}$ Supportive Center for Brain Research, National Institute for Physiological Science, Okazaki 444-8585, Japan, ${ }^{4}$ PRESTO, Japan Science and Technology Agency (JST), Kawaguchi 332-0012, Japan, ${ }^{5}$ Department of Neurophysiology, Hamamatsu University School of Medicine, Hamamatsu 431-3192, Japan, 'Department of Neurophysiology, Kagawa School of Pharmaceutical Sciences, Tokushima Bunri University, Tokushima 769-2193, Japan, ${ }^{7}$ Division of Molecular and Developmental Biology, National Institute of Genetics, Mishima 411-8540, Japan, ${ }^{8}$ Department of Genetics, The Graduate University for Advanced Studies (SOKENDAI), Mishima 411-8540, Japan, ${ }^{9}$ Department of Chemistry and Biological Science, Graduate School of Science and Engineering, Aoyama Gakuin University, Sagamihara 252-5258, Japan, ${ }^{10}$ Department of Physiology, School of Medical Sciences, University of New South Wales, Sydney 2052, Australia, ${ }^{11}$ Department of Physiology, Kitasato University School of Allied Health Sciences, Sagamihara 252-0373, Japan, and ${ }^{12}$ CREST, Japan Science and Technology Agency (JST), Kawaguchi 332-0012, Japan
\end{abstract}

\begin{abstract}
Inhibitory synapses are established during development but continue to be generated and modulated in strength in the mature nervous system. In the spinal cord and brainstem, presynaptically released inhibitory neurotransmitter dominantly switches from GABA to glycine during normal development in vivo. While presynaptic mechanisms of the shift of inhibitory neurotransmission are well investigated, the contribution of postsynaptic neurotransmitter receptors to this shift is not fully elucidated. Synaptic clustering of glycine receptors (GlyRs) is regulated by activation-dependent depolarization in early development. However, GlyR activation induces hyperpolarization after the first postnatal week, and little is known whether and how presynaptically released glycine regulates postsynaptic receptors in a depolarization-independent manner in mature developmental stage. Here we developed spinal cord neuronal culture of rodents using chronic strychnine application to investigate whether initial activation of GlyRs in mature stage could change postsynaptic localization of GlyRs. Immunocytochemical analyses demonstrate that chronic blockade of GlyR activation until mature developmental stage resulted in smaller clusters of postsynaptic GlyRs that could be enlarged upon receptor activation for $1 \mathrm{~h}$ in the mature stage. Furthermore, live cell-imaging techniques show that GlyR
\end{abstract}

\section{Significance Statement}

Clustering of postsynaptic glycine receptors (GlyRs) is critical for the developmental shift from GABAergic to glycinergic inhibitory neurotransmission in the spinal cord and brainstem. Synaptic GlyR localization is mediated by the receptor activation and followed by depolarization-dependent $\mathrm{Ca}^{2+}$ influx in the immature stage. However, little is known whether and how presynaptically released glycine enhances postsynaptic GlyR clustering in the mature stage when glycinergic transmission is upregulated without depolarization. Here we report postsynaptic GlyR clustering induced by the receptor activation in mature neurons. Furthermore, postsynaptic stabilization of laterally diffusive GlyRs is mediated in $\mathrm{Ca}^{2+}$-independent PKC activity at sites expressing gephyrin, a postsynaptic scaffolding protein. Our results provide new insights into homeostatic receptor dynamics underlying activation-dependent modulation of inhibitory synaptic strength in the mature nervous system. 
activation decreases its lateral diffusion at synapses, and this phenomenon is dependent on PKC, but neither $\mathrm{Ca}^{2+}$ nor CaMKII activity. These results suggest that the GlyR activation can regulate receptor diffusion and cluster size at inhibitory synapses in mature stage, providing not only new insights into the postsynaptic mechanism of shifting inhibitory neurotransmission but also the inhibitory synaptic plasticity in mature nervous system.

Key words: gephyrin; glycine receptor (GlyR); inhibitory synaptic plasticity; mature neuron; spinal cord; synaptic clustering

\section{Introduction}

GABA and glycine mediate fast inhibitory neurotransmission in the mature nervous system by activating postsynaptic $G A B A_{A}$ and glycine receptors, respectively, to reduce neuronal excitability via postsynaptic hyperpolarization and membrane shunting. The inhibitory neurotransmissions in the adult brainstem and spinal cord are predominantly mediated by glycine and play essential roles for the auditory system and the ascending nociceptive pathways (Sanes et al., 1998; Lynch and Callister, 2006). Interestingly, the predominant neurotransmission during the early developmental stage is GABAergic and developmentally shifts to glycinergic in these synapses (Kotak et al., 1998; Gao et al., 2001; Russier et al., 2002). This unique developmental feature is primarily regulated by switching the proportions of GABA and glycine in presynaptic vesicles (Nabekura et al., 2004). In the presynaptic terminals, vesicular inhibitory amino acid transporter [VIAAT (or VGAT)] uptakes both GABA and glycine with different binding affinity (McIntire et al., 1997); thus, the relative proportions of presynaptic glycine and GABA concentrations determine the contents of released inhibitory neurotransmitters (Ishibashi et al., 2013). Therefore, with developmental reductions of glutamic acid decarboxylases, GABA-synthesizing enzymes regulate the switching of presynaptically released inhibitory neurotransmitters (Ma et al., 1994; Nabekura et al., 2004).

Received July 6, 2016; accepted January 17, 2017; First published January 25, 2017

The authors declare no competing financial interests.

Author contributions: Y.N., H.M., H.I., and J.N. designed research; Y.N., M.W., T.K., and H.H. performed research; H.M contributed unpublished analytic tools; Y.N., and M.W. analyzed data; Y.N., K.E, A.J.M., and J.N. wrote the paper.

This work was supported by Grants-in-Aid for Scientific Research (KAKENHI) from the Japan Society for the Promotion of Science to Y.N. (11J02839, 14J30003, and 26870826) and to J.N. (25253017).

Acknowledgments: We thank Dr. H. Bannai (Nagoya University, Nagoya, Japan), Dr. T. Inoue (Waseda University, Tokyo, Japan), Dr. A. Triller (Ecole Normale Supérieure, Paris, France), and Dr. K. Mikoshiba (RIKEN Brain Science Institute, Wakō City, Japan) for providing technical skills and software for single-particle tracking. We also thank Dr. Y. Fukata and Dr. M. Fukata (National Institute for Physiological Sciences, Okazaki, Japan) for expert technical assistance; Dr. Y. Fukazawa (University of Fukui, Fukui, Japan); and Dr. G. Miesenböck (University of Oxford, Oxford, UK) for providing the SEP construct. In addition, we thank the Spectrography and Bioimaging Facility, National Institute for Basic Biology Core Research Facilities (Okazaki, Japan) for confocal microscopy facilities.

Correspondence should be addressed to Junichi Nabekura, Division of Homeostatic Development, Department of Developmental Physiology, National Institute for Physiological Sciences, 38 Nishigonaka, Myodaiji, Okazaki 444-8585, Japan. E-mail: nabekura@nips.ac.jp.

DOI:http://dx.doi.org/10.1523/ENEURO.0194-16.2017

Copyright (C) 2017 Nakahata et al.

This is an open-access article distributed under the terms of the Creative Commons Attribution 4.0 International, which permits unrestricted use, distribution and reproduction in any medium provided that the original work is properly attributed.
Although the presynaptic mechanism underlying the developmental shift of inhibitory neurotransmission has been well investigated, it is still has not been elucidated whether and how postsynapses contribute to the shift of inhibitory neurotransmission. The postsynaptic localization of appropriate neurotransmitter receptors is primarily essential for the effective neurotransmission, and two scenarios in postsynaptic receptor localization are postulated for the developmental shift of neurotransmission. One scenario is that both $\mathrm{GABA}_{\mathrm{A}}$ receptors and glycine receptors (GlyRs) are preexisting and stable; therefore, the developmental shift of neurotransmission is purely attributable to the proportions of presynaptically released GABA/glycine. The other scenario is that postsynaptic receptor localization is actively modulated in correspondence with developmental switching of released neurotransmitters. The number of receptors clustering postsynaptically is determined first by trafficking of the receptors between the cytoplasm and the plasma membrane at extrasynaptic sites (exocytosis and endocytosis), and, second, by the lateral diffusion of receptors in the plasma membrane and their stabilization at synaptic sites (Jacob et al., 2008). Extensive studies on excitatory synapses indicate that neurotransmission-dependent stabilization of laterally diffusing postsynaptic receptors may be a principal determinant of excitatory synaptic potentiation (Huganir and Nicoll, 2013). Similarly, the inactivation of postsynaptic GlyRs suppressed formation of the receptor clusters during early development in spinal cord neurons (Kirsch and Betz, 1998; Lévi et al., 1998), indicating that the neurotransmitter itself promotes receptor clustering. Thus, the neurotransmission-dependent clustering of GlyRs was explained by the GlyR-mediated depolarization and subsequent $\mathrm{Ca}^{2+}$ influx and CaMKII activation that occur when GlyRs are activated in early development (Kirsch and Betz, 1998; Yamanaka et al., 2013).

$\mathrm{GABA}_{\mathrm{A}}$ receptor- or GlyR-mediated neurotransmissions developmentally shift from depolarizing to hyperpolarizing responses (D-H shift) after the first postnatal week with decreasing intracellular $\mathrm{Cl}^{-}$concentration (Kakazu et al., 1999; Ben-Ari, 2002). However, the expression of GlyR $\alpha 1$, the mature-type subunit constructs the heteromeric GlyR, which localizes at postsynapses, and increases in parallel with the upregulated presynaptic glycine releases, and this persists after the D-H shift up to at least the third postnatal week (Friauf et al., 1997; Korada and Schwartz, 1999). Therefore, the activationinduced depolarization model in early development may be insufficient to explain the postsynaptic clustering of GlyRs in the mature stage, and we hypothesize that the postsynaptic localization of GlyRs are regulated by presynaptically released glycine in a depolarization- 
independent manner in mature stage. To address this question, we generated a spinal neuron culture model to distinguish and mimic the initial activation events of GlyRs after the D-H shift. In this model, we chronically prevented activation of GlyRs by strychnine, a competitive GlyR antagonist treatment, during the developmental period. Then GlyRs are activated after the D-H shift by strychnine removal. Thus, this model enables us to analyze the effects of GlyR activation on the spatiotemporal dynamics of postsynaptic GlyRs in mature neurons. In contrast to the current depolarization-dependent model of GlyR clustering, we found that the activation of GlyRs in more mature neurons could still elicit changes in diffusion and increases in the postsynaptic GlyR clusters.

\section{Materials and Methods}

All procedures performed in this study were approved by the Okazaki Institutional Animal Care and Use Committee and were conducted in accordance with guidelines defined by the National Institute of Natural Sciences. All efforts were made to minimize the suffering and number of animals used in this study.

\section{Primary spinal cord culture}

Dissociated primary cultures of spinal cord neurons were prepared as described previously (Ishibashi et al., 2013). Wild-type C57BL/6 mice (Japan SLC) were used for electrophysiology and experiments with DNA transfection, VGAT-Venus transgenic C57BL/6 mice (Wang et al., 2009) were used for quantum dot (QD) single-particle tracking (SPT) and wild-type Wistar rats (Japan SLC) were used for immunocytochemistry and immunoblotting. Briefly, embryos [embryonic day 13 (E13) to E15] of both sexes were isolated from pregnant animals under isoflurane anesthesia and were immediately placed into icecold Leibovitz's L-15 medium (Life Technologies). Spinal cords were quickly removed and incubated in HEPESbuffered DMEM containing papain ( $24 \mu \mathrm{l} / \mathrm{ml}$; Worthington) for $20 \mathrm{~min}$ at $32^{\circ} \mathrm{C}$. Individual cells were dissociated by repeated gentle pipette trituration in Neurobasal medium containing DNase and L-glutamate. Neurons were collected by centrifugation at $8000 \mathrm{rpm}$ for $1 \mathrm{~min}$, and the supernatant was exchanged with fresh Neurobasal medium containing B27 supplement $(50 \times)$ ), Glutamax (Life Technologies), penicillin $100 \mathrm{U} / \mathrm{ml}$, and streptomycin 100 $\mu \mathrm{g} / \mathrm{ml}$. Cells were plated on polyethylenimine-coated coverslips, either $18 \mathrm{~mm}$ diameter or $8 \times 8 \mathrm{~mm}$ (Matsunami) at a density of 1.0-1.6 $\times 10^{4}$ cells $/ \mathrm{cm}^{2}$, and were incubated at $37^{\circ} \mathrm{C}$ and $5 \% \mathrm{CO}_{2}$ for $14-42 \mathrm{~d}$ before the experiments.

Three experimental groups were typically used (see Fig. $2 A)$. The first group [chronic strychnine (cSTR)] was chronically treated with strychnine $(1 \mu \mathrm{M})$ to block GlyR activation from the first day of culture through to when experiments were performed after at least 2 weeks in culture, when neurons and their intracellular $\mathrm{Cl}^{-}$concentrations were equivalent to mature neurons. The second group was similarly treated with chronic administration of strychnine, but strychnine was then washed out $1 \mathrm{~h}$ prior to each experiment (WASH group). Hence, neurons in this group had GlyRs activated by endogenous synaptic ac- tivity for $1 \mathrm{~h}$. The third group [control (Ctrl)] was treated with vehicle (Neurobasal culture medium, the solvent of strychnine) from the first day of culture plating, resulting in normal activation of GlyRs throughout development from endogenous synaptic activity.

\section{DNA Constructs and transfection}

The coding sequence of superecliptic pHluorin (SEP; a gift from Dr. G. Miesenböck, University of Oxford, Oxford, UK) was fused at the extracellular domain of the mouse GlyR $\alpha 1$ subunit, just following the signal peptide sequence (pCAG-SEP-GlyR $\alpha 1$ ). The mCherry-gephyrin (GPHN) construct was constructed from the rat gephyrin-P1 splice variant 1 , which was labeled at its $\mathrm{N}$ terminus with mCherry (Kuriu et al., 2012). Cells were transfected 13-15 d in vitro (DIV) and imaged within $48 \mathrm{~h}$ after transfections. Transfections were performed by incubating cell in Lipofectamine 2000 (Life Technologies) in Neurobasal medium lacking penicillin-streptomycin for $6-8 \mathrm{~h}$.

\section{Reagents}

Drugs used in the present study were $5 \mu \mathrm{M}$ DL-2-amino-7phosphonovalerate (DL-APV), $5 \mu \mathrm{M}$ 6-cyano-7-nitroquinoxaline-

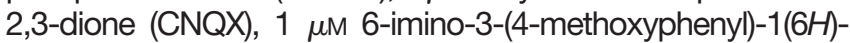
pyridazinebutanoic acid (SR95531), $1 \mu \mathrm{M}$ strychnine, 20-40 $\mu \mathrm{g} / \mathrm{ml}$ gramicidin D, $100 \mu \mathrm{M}$ or $1 \mathrm{M}$ glycine, $5 \mu \mathrm{g} / \mathrm{ml}$ brefeldin A (BFA), $1 \mu \mathrm{m}$ ginkgolide $\mathrm{B}, 0.5 \mu \mathrm{m}$ mecamylamine hydrochloride, $10 \mu \mathrm{M}$ BAPTA-AM, $30 \mu \mathrm{M}$ KN-62, $10 \mu \mathrm{M}$ Rp-adenosine 3, and $1 \mu \mathrm{M}$ ginkgolide monophosphorothioate triethylammonium salt (all from Sigma-Aldrich Japan); 50 nм GF 109203X (GFX; Enzo Life Sciences); and $0.3 \mu \mathrm{M}$ tetrodotoxin (TTX; Latoxan). Drugcontaining solutions were locally applied via a Y-tube perfusion system (Murase et al., 1989) in electrophysiological recordings and via bath application in imaging experiments.

\section{Solutions}

The standard extracellular solution for imaging and electrophysiological experiments contained the following (in $\mathrm{mm}$ ): $148 \mathrm{NaCl}, 5 \mathrm{KCl}, 1 \mathrm{MgCl}_{2}, 2 \mathrm{CaCl}_{2}, 10$ glucose, and 10 HEPES, and adjusted to $\mathrm{pH} 7.4$ with Tris-OH. In all experiments, the standard extracellular solution contained the (in $\mu \mathrm{M}$ ) 5 CNQX, 5 DL-APV, 1 SR95531, and 0.3 TTX, concentrations that were sufficient to fully inhibit spontaneous excitatory and GABAergic postsynaptic currents, and action potentials. Additionally, $1 \mu \mathrm{M}$ strychnine was constantly present in the cSTR group and was present in the WASH group until $1 \mathrm{~h}$ before experiments. QD binding buffer contained the following (in $\mathrm{mm}$ ): 2.62 sodium tetraborate decahydrate, 39.5 boric acid, and $2 \%$ (w/v) bovine serum albumin and adjusted to $\mathrm{pH} 7.4$ with 1 $\mathrm{M} \mathrm{HCl}$ or $1 \mathrm{M} \mathrm{NaOH}$. In electrophysiological experiments, the internal pipette solution for the perforated patchclamp configuration contained the following (in $\mathrm{mm}$ ): 90 $\mathrm{KCl}, 60 \mathrm{~K}-\mathrm{methanesulfonate}$, and $10 \mathrm{HEPES}$. The pipette solution for conventional whole-cell configuration contained the following (in $\mathrm{mm}$ ): $100 \mathrm{~K}$-methanesulfonate, 8 $\mathrm{NaCl}, 30 \mathrm{KCl}, 1 \mathrm{MgCl}_{2}$, 2 EGTA, 4 Mg-ATP, 5 QX-314-Cl, and 10 HEPES. All pipette solutions were adjusted to $\mathrm{pH}$ 7.3 with Tris-OH. In a perforated patch-clamp configuration, gramicidin D was initially dissolved in DMSO at 10 $\mathrm{mg} / \mathrm{ml}$ and then diluted to a final concentration of $20-40$ 
$\mu \mathrm{g} / \mathrm{ml}$ in the pipette solution. The gramicidin was prepared just before use.

\section{Electrophysiological measurements}

Cultured neurons were observed under phase contrast on an inverted microscope (model IX70, Olympus). Ionic currents were measured with a Multiclamp 700B patchclamp amplifier (Molecular Devices) and were recorded with a sampling frequency of $20 \mathrm{kHz}$ after low-pass filtering at $5 \mathrm{kHz}$. Currents were recorded, and voltage protocols were applied, using pClamp 10.2 software (Molecular Devices; RRID: SCR_011323). The resistance between the patch pipette filled with the internal solution and the reference electrode in the standard external solution was 6-8 $\mathrm{M} \Omega$. The neurons were voltage clamped at a holding potential of $-53 \mathrm{mV}$ in gramicidin-perforated patch experiments and $-70 \mathrm{mV}$ in the conventional whole-cell patch-clamp configurations.

The reversal potentials of glycine-induced currents $\left(E_{\text {gly }}\right)$ were recorded using gramicidin-perorated patch configurations to maintain intracellular $\mathrm{Cl}^{-}$concentrations intact (Ebihara et al., 1995). Voltage-ramps from -113 to $+37 \mathrm{mV}$, with duration of $1.6 \mathrm{~s}$, were applied to the neuron before and during glycine application. Voltage protocols were generated by a software-driven digital-to-analog converter (Digidata 1322A, Molecular Devices). Corrections for the liquid junction potentials $(-3.4 \mathrm{mV})$ were applied to the data in gramicidin-perforated recordings. Current-voltage $(I-V)$ relationships were measured by subtracting current values during the control from those during glycine application, and the $E_{\text {gly }}$ was quantified as the reversal potential of this control subtracted current.

Spontaneous mIPSCs were recorded using a conventional whole-cell patch-clamp configuration. To measure mIPSCs, in the cSTR group the bath solution including strychnine was completely exchanged with the standard recording solution lacking strychnine immediately before recording. In the control group, the bath solution was exchanged to a recording solution that included $1 \mu \mathrm{M}$ strychnine for $30 \mathrm{~s}$ and then was exchanged again to the standard solution lacking strychnine in order to exclude the effect of any residual strychnine between the control and chronic strychnine treatment groups. Recordings started within 10 min after strychnine washout and lasted for $60 \mathrm{~min}$. mIPSCs were confirmed by application of $1 \mu \mathrm{M}$ strychnine after each recording. All experiments were performed at room temperature $\left(21-24^{\circ} \mathrm{C}\right)$. Recordings were discontinued if the access resistance changed $>30 \%$.

Spontaneous mIPSCs were analyzed using the template search tool in Clampfit 10.2 (Molecular Devices). The template was created from 100 responses and automatically detected events. Amplitude threshold values were set at threefold of SD values of the baseline noise amplitude. False-positive results were rejected by visual inspection. Over 200 events were detected and statistically analyzed at each time point $\pm 2.5 \mathrm{~min}$ from each neuron. Decay time constants were calculated by fitting a doubleexponential function to the mIPSC decay from the time period corresponding to that between $90 \%$ and $10 \%$ of the peak mIPSC amplitude.

\section{Immunostaining, image acquisition, and quantitative analysis}

Cultured rat spinal cord neurons were fixed with paraformaldehyde (PFA; 4\% w/v) in PBS and then permeabilized with Triton X-100 $(0.1 \% \mathrm{v} / \mathrm{v})$. Nonspecific antibodybinding sites were blocked with PBS containing normal goat serum $(2 \% \mathrm{v} / \mathrm{v})$ and bovine serum albumin $(1 \% \mathrm{w} / \mathrm{v})$. Cells were double labeled using the following primary antibodies: polyclonal rabbit anti-VIAAT (1:500; Synaptic Systems; RRID: AB_887871), polyclonal guinea pig antiVIAAT (1:1000; catalog \#131-004, Synaptic Systems; RRID: AB_887873), monoclonal mouse anti-glycine receptor $\alpha 1$ (mAb2b; 1:200; Synaptic Systems 146-111; RRID: AB_887723), and polyclonal rabbit anti-gephyrin (1:1000; catalog \#147-003, Synaptic Systems; RRID: $A B \_887718$ ) overnight at $4^{\circ} \mathrm{C}$, followed by incubation with fluorescence-conjugated secondary antibodies (Alexa Fluor 488, RRID: AB_2534117 or AB_2534069, 1:1000; Alexa Fluor 594, RRID: AB_2534095, 1:1000; Alexa Fluor 633, RRID: AB_2535731, 1:1000; Life Technologies) for 30 $\mathrm{min}$ at room temperature. Coverslips were mounted in PermaFluor aqueous mounting medium (Thermo Shandon). Fluorescence images were acquired with a confocal laser-scanning microscope [Plan Apo-VC $100 \times \mathrm{H}$, numerical aperture (NA) 1.4; model A1, Nikon]. Pixel size and focus steps were 0.1 and $0.37 \mu \mathrm{m}$, respectively, with images of $1024 \times 1024$ pixels.

The number and areas of puncta were measured using MetaMorph 7.7 (Molecular Devices) as described previously (Kuriu et al., 2012), with slight modifications. Maximal intensity projection images were prepared for each image stack, and these projection images were filtered using a $3 \times 3$ pixel low-pass filter to remove noise. Images were converted to binary images using an intensity threshold to determine the outline of the punctate signals. After applying built-in erosion and dilation processing to remove single-pixel noises, the numbers and areas of fluorescence signals were automatically counted from binary images using the integrated morphometry analysis function. Colocalization of immunostained presynaptic marker proteins (VIAAT) and postsynaptic GlyRs was determined using Wright Cell Imaging Facility ImageJ software (RRID: SCR_008488) based on the overlap coefficient of Manders et al. (1993) after automated background subtraction to avoid user bias in setting analysis parameters. A colocalization coefficient value of 0 indicates nonoverlapping immunostained proteins, while a coefficient of 1 corresponds to $100 \%$ colocalization. The coefficient indicates the proportion of the GlyR or GPHN signals coincident with the VIAAT signal intensity. Results were shown as the ratio of the summed intensities of pixels from the GlyR or GPHN for which the intensity of VIAAT was above zero.

\section{Cell surface biotinylation and immunoblotting}

Surface biotinylation experiments were performed using a cell surface protein biotinylation and purification kit (ThermoFisher Scientific) according to the manufacturer 
protocol. Briefly, cultured spinal cord neurons from four individual cultures (19-33 DIV) were washed with ice-cold PBS and then labeled with $0.25 \mathrm{mg} / \mathrm{ml}$ sulfo-NHS-SSbiotin for 30 min at $4^{\circ} \mathrm{C}$ before washing with PBS supplemented with quenching solution to remove excess biotin. The cell lysates were centrifuged $(10,000 \times g$, for $2 \mathrm{~min})$, the supernatant was isolated with NeutrAvidin gel, and the bound proteins were then eluted with SDS sample buffer (62.5 mM Tris- $\mathrm{HCl}, \mathrm{pH} 6.8,1 \%$ SDS, and 10\% glycerol) and were analyzed by SDS-PAGE and Western blotting.

Proteins were separated using $7.5 \%$ acrylamide gels by SDS-PAGE. The gels were transferred to an Immobilon-P membrane (Millipore). The blots were blocked in 1\% bovine serum albumin and incubated overnight with primary antibody at $4^{\circ} \mathrm{C}$. They were then incubated with horseradish peroxidase-conjugated secondary antibody (GE Healthcare UK) for $1 \mathrm{~h}$ at room temperature. Enhanced chemiluminescence (ECL; GE Healthcare) exposure on instant film and an ECL mini-camera luminometer were used to visualize labeled protein. The following primary antibodies were used: anti-pan GlyRs (1:1000; mAb4a, Synaptic Systems; RRID: AB_887722) and anti-actin (1: 10,000; Sigma-Aldrich- RRID: AB_476744). The optical densities of bands were analyzed with ImageJ software (RRID: SCR_003070). Since pan-GlyR antibody (mAb4a) detects isoforms of GlyR $\alpha$ subunits, we analyzed the density of assumptive bands indicating 48 kDa GlyR $\alpha 1$ (Becker et al., 1992; Weltzien et al., 2012). The densities of the total GlyR band and the surface GlyR band were normalized to the densities of actin band and total band, respectively.

\section{Fluorescence recovery after photobleaching and fluorescence loss in photobleaching}

For experiments applying fluorescence recovery after photobleaching (FRAP) and fluorescence loss in photobleaching (FLIP; Jaskolski et al., 2009; Hildick et al., 2012), mouse spinal cord neurons (13-15 DIV) were transfected with pCAG-SEP-GlyR $\alpha 1$ (and CMV-mCherrygephyrin) constructs at $24-48 \mathrm{~h}$ prior to experiments. Imaging was performed at room temperature $\left(24 \pm 2^{\circ} \mathrm{C}\right)$ in the standard recording solution, and images were acquired with a confocal laser-scanning microscope (Plan Apo-VC $60 \times$ H, NA 1.4; model A1, Nikon). Fluorescence of SEP was photobleached using a $488 \mathrm{~nm}$ laser at a full power of $40 \mathrm{~mW}$ for $62.5 \mathrm{~ms}$, repeated seven times in a 3 $\times 5 \mu \mathrm{m}$ rectangular region of interest (ROI) in SEPexpressed neurites. For the focal FRAP protocol, photobleaching was applied to two $1-\mu \mathrm{m}$-diameter circular regions of interests with and without mCherry-expressing puncta. Laser power at $1 \%$ was used for image acquisitions. In the FRAP-FLIP protocol, repetitive photobleaching at the FLIP regions ( $3 \times 5 \mu \mathrm{m}$ rectangles) was applied throughout the imaging period directly lateral to a larger initial central bleaching region $(10 \times 5 \mu \mathrm{m})$. The fluorescence intensity of the central ROI $(3 \times 5 \mu \mathrm{m})$ was measured. Since the fluorescence intensity of SEP can be observed in early endosomes, the baseline intensity before photobleaching may contain this fluorescence intensity. Thus, we confirmed surface fractions of SEP fluorescence by extracellular acidification ( $\mathrm{pH}$ 5.5), which quenches fluorescence after FRAP experiments. In addition, fluorescence in early endosome fractions is photobleached in the ROI; thus, it may not affect fluorescence recovery after photobleaching.

Images were acquired 93 times with $2.9 \mathrm{~s}$ intervals in wide FRAP and FRAP-FLIP experiments, and 93 times with $3 \mathrm{~s}$ in focal FRAP experiments. The intensity of fluorescence recovery was measured after background subtraction and a correction based on the intensity of the unbleached regions, and was normalized to prebleach intensity. The average fluorescence intensities after photobleaching were fitted by single exponential recovery curves using KaleidaGraph 4.1 (HULINKS). The mobile fraction (MF) was calculated according to Equation 1:

$$
\mathrm{MF}=\left(F_{\max }-F_{(0)}\right) /\left(F_{\text {pre }}-F_{(0)}\right)
$$

where $F_{\text {pre }}$ is the prebleach intensity, $F_{\max }$ is the plateau intensity after photobleaching, and $F_{(0)}$ is the postbleach intensity at time zero.

For the experiment monitoring fluorescence intensity under local application of glycine, the standard extracellular solution containing both $1 \mathrm{~m}$ glycine and Alexa Fluor 594 hydrazide (ThermoFisher Scientific) is applied to single dendrites by glass pipettes (approximate tip diameter, $1 \mu \mathrm{m})$ at $2 \mathrm{~Hz}$ using a valve-controlled pressure application system (IM300 Microinjector). The area of local glycine application was confirmed by monitoring the fluorescence of Alexa Fluor 564 hydrazide. The fluorescence intensity of SEP-GlyR $\alpha 1$ was measured at gephyrin-positive $\left(\mathrm{GPHN}^{+}\right)$ and gephyrin-negative $\left(\mathrm{GPHN}^{-}\right)$dendritic shafts, which were covered by glycine application.

\section{Quantum dot live cell staining, image acquisition, and quantitative analysis}

After a single wash with Neurobasal medium, neurons were incubated for 5 min with a primary antibody that recognizes an extracellular epitope of the GlyR $\alpha 1$ subunit (5 $\mu \mathrm{g} / \mathrm{ml}$; mAb2b, Synaptic Systems; RRID: AB_887723), then neurons were washed five times with Neurobasal medium. They were subsequently incubated for $5 \mathrm{~min}$ with the secondary antibody $[5.2 \mu \mathrm{g} / \mathrm{ml}$; biotin Fab goat anti-mouse IgG $(\mathrm{H}+\mathrm{L})$, Jackson ImmunoResearch; RRID: $\left.A B \_2338586\right]$, then washed again five times. The neurons were next incubated with streptavidin-conjugated quantum dots emitted at $605 \mathrm{~nm}$ (1 nM; Life Technologies) in QD binding buffer (Bannai et al., 2006) for 1 min before washing eight times with the standard extracellular solution. Finally, neurons were incubated with FM4-64 (2 $\mu \mathrm{M}$; Life Technologies) in $40 \mathrm{~mm} \mathrm{KCl}$ standard solution for 30 $\mathrm{s}$ to label active presynaptic sites (Gaffield and Betz, 2006), before returning to the standard external solution. QD labeling, washing, and image acquisition procedures were performed at $37^{\circ} \mathrm{C}$

QD-labeled neurons were imaged in the standard recording solution using an inverted microscope (model IX70, Olympus) equipped with an oil-immersion objective (100×, NA 1.4), and an EM-CCD camera (ImagEM C910013, Hamamatsu Photonics), mercury lamp, appropriate bandpass filter sets for QD 605 (excitation wavelength 
(ex.) 457/50 and emission wavelength (em.) 605/15), FM4-64 (ex. 510/84 and em.736/128), and Venus (ex. $457 / 50$ and em.530/55). Single images of FM4-64 were taken before the QD imaging. QD image acquisition movies were composed of 300 frames, with each frame being of $67 \mathrm{~ms}$ duration and separated by $33 \mathrm{~ms}$, giving a total imaging time of $30 \mathrm{~s}$ with MetaMorph software version 7.7 (Molecular Devices; RRID: SCR_002368). The movements of QD-GlyR $\alpha 1$ s were recorded within 45 min after labeling. Strychnine $(1 \mu \mathrm{M})$ was presented only in the cSTR group during staining and imaging.

For single-particle tracking, QD-GlyR particles on Venus-expressing neurons were selectively analyzed. The trajectory of each particle was determined by crosscorrelating the image with a Gaussian model of the point spread function (Bonneau et al., 2005), using TI workbench software written by $\mathrm{T}$. Inoue (Waseda University, Tokyo, Japan; Bannai et al., 2009). Only single QDs identified by their characteristic blinking were analyzed, and particles that fluoresced in >295 (of 300) frames were excluded. The synaptic regions were defined by processing FM4-64 images with top-hat filtering (Waataja et al., 2008). For each frame, QD-GlyR fluorescence that merged by at least 1 pixel with the binarized image of FM 4-64 was defined as a synaptic location, and if the QD was $>2$ pixels away from the FM 4-64 image, this was defined as extrasynaptic. For the calculation of diffusion parameters in the synaptic and extrasynaptic regions, the longest subtrajectories (segments) of single QD-GlyRs that consisted of at least 30 frames in each compartment were used to represent the behavior of each particle in that compartment. Synaptic dwell times were quantified by counting the total number of consecutive frames in which a QD was localized to synaptic regions.

To obtain the diffusion coefficients, values of the mean square displacement (MSD) plot versus time were calculated for each trajectory using Equation 2 (Saxton and Jacobson, 1997), as follows:

$$
\begin{aligned}
M S D(n \tau)= & \frac{1}{N-n} \sum_{i=1}^{N-n}\left[(x((i+n) \tau)-x(i \tau))^{2}\right. \\
& \left.+(y((i+n) \tau)-y(i \tau))^{2}\right]
\end{aligned}
$$

where $\tau$ is the acquisition time, $N$ is the total number of frames, and $n$ and $i$ are positive integers with $n$ representing the time increment. Diffusion coefficients $(D)$ were calculated by fitting the first three points of the MSD versus time curves with Equation 3 (Kusumi et al., 1993; Bannai et al., 2006), as follows:

$$
M S D(n \tau)=4 D n \tau+b
$$

where $b$ is a constant reflecting the spot localization accuracy.

The confinement sizes $(L)$ were calculated by fitting the MSD- $n \tau$ plot of the trajectories showing a restricted motion to Equation 4 (Kusumi et al., 1993), as follows:

$$
M S D(n \tau)=\frac{L^{2}}{3}\left(1-\exp \left(-\frac{12 D n \tau}{L^{2}}\right)\right)+4 D_{m a c} n \tau
$$

where $L^{2}$ is the confinement area where diffusion is restricted, and $D_{\text {mac }}$ is the diffusion coefficient on a long time scale.

\section{Statistical analysis}

Data are presented as the median \pm interquartile range for diffusion coefficients obtained from SPT. All other data are expressed as the mean \pm SEM. Statistical significance was determined using Student's unpaired twotailed $t$ test for comparing the reversal potential of glycineinduced currents, for comparing the decay time constant of mIPSCs and for comparing changes in SEP-GlyR $\alpha 1$ fluorescence intensities at gephyrin-positive and gephyrinnegative areas by local glycine application; Student's paired two-tailed $t$ test for comparing fractions of fluorescence recovery between mCherry-GPHN and SEPGlyR $\alpha 1$; one-way ANOVA with Bonferroni's post hoc test for multiple comparisons for immunostaining experiments and for dwell time and confinement size in SPT experiments; and Kruskal-Wallis test followed by post hoc comparisons using Mann-Whitney $U$ test with Bonferroni's correction for other statistical comparisons. Pearson's correlation coefficient was calculated using linear regression fit on the fractions of fluorescence recovery between mCherry-GPHN and SEP-GlyR $\alpha 1$.

\section{Results}

\section{Activation-dependent increase in the amplitude of postsynaptic glycine responses}

The present study used a culture model in which initial activation of GlyRs could be pharmacologically delayed until after the developmental shift from depolarizing to hyperpolarizing responses (Fig. 1A). Washout of strychnine, which had been applied from the first DIV until each experiment, allowed initial activation of GlyRs once neurons had matured. To validate this model, we first examined whether the cSTR application $(1 \mu \mathrm{M})$ affected the typical maturation of intracellular $\mathrm{Cl}^{-}$concentrations. We cultured primary spinal cord neurons over 14 DIV and measured the reversal potential of $E_{\text {gly }}$ using gramicidinperforated patch-clamp recording (Fig. 1C). By subtracting the amplitude of currents that were induced by ramp pulses between the presence and absence of $100 \mu \mathrm{M}$ glycine (Fig. 1C), the $I-V$ curves were obtained (Fig. 1D). The reversal potentials in cSTR conditions $(-70.6 \pm 1.9$ $\mathrm{mV}, n=5$ ) were comparable to those in the nonstrychnine Ctrl conditions $(-74.2 \pm 0.8 \mathrm{mV} ; n=6 ; p=0.25$; Fig. 1D). These results indicate that chronic treatment with strychnine does not affect the development of the intracellular $\mathrm{Cl}^{-}$regulatory system, and that glycinergic transmission after a 2 week culture induces hyperpolarization.

To examine whether the initial activation of postsynaptic GlyRs in the mature stage subsequently modulates glycinergic transmission, we chronically incubated cultures with $1 \mu \mathrm{M}$ strychnine, from the first DIV until immediately before the experiment (>14 DIV; Fig. 1B). Spontaneous mIPSCs were measured by the conventional whole-cell patch-clamp recording in the presence of TTX, CNQX, DL-APV, and SR95531 in the standard bath 
A

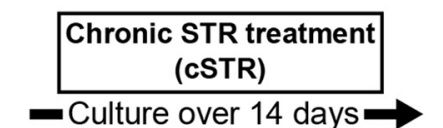

Gly

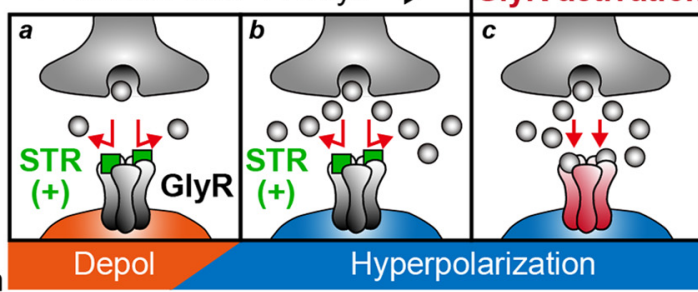

C

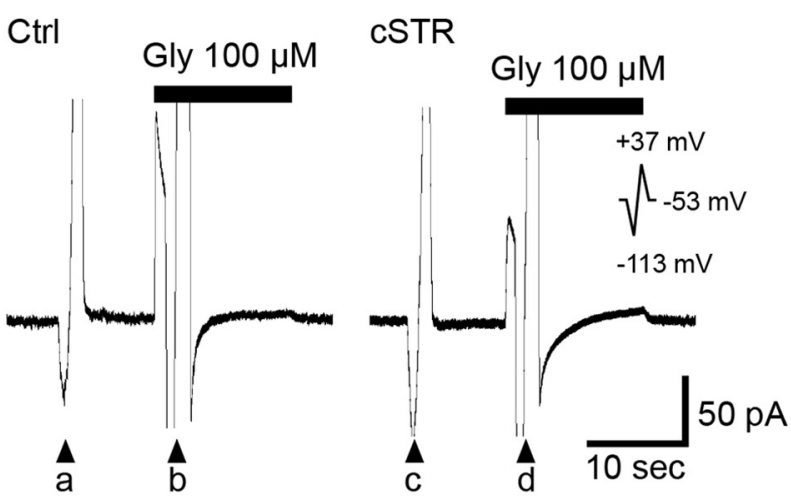

TR removal (WASH)

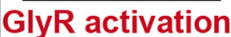

GlyR activation
B

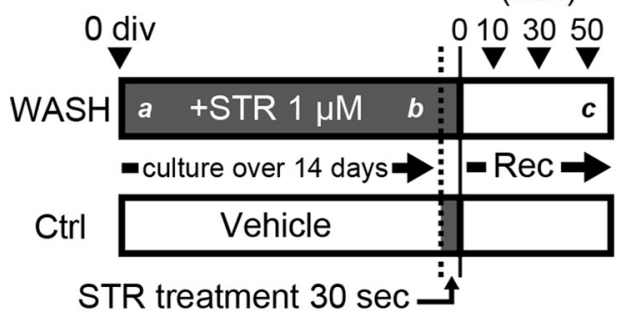

D

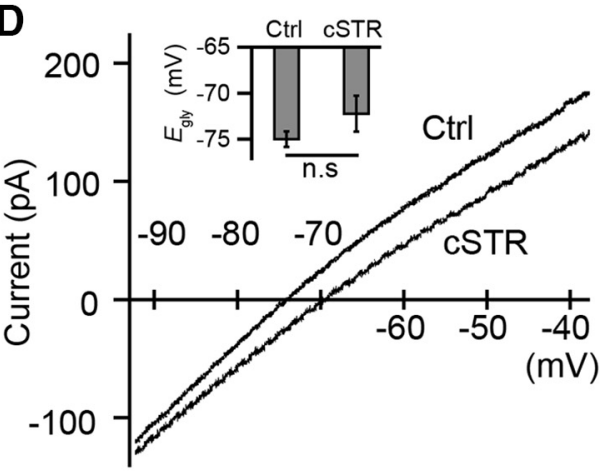

E
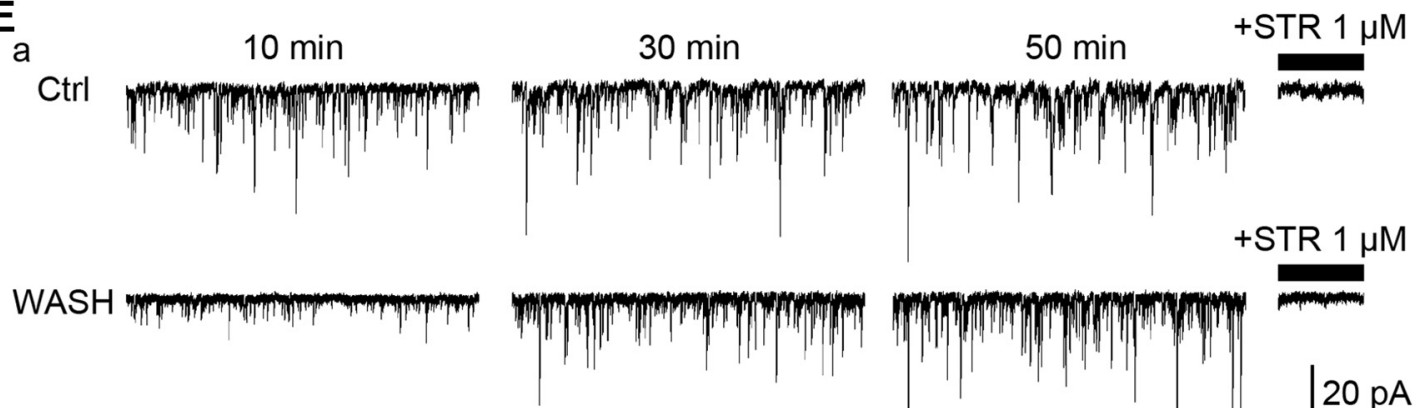

b
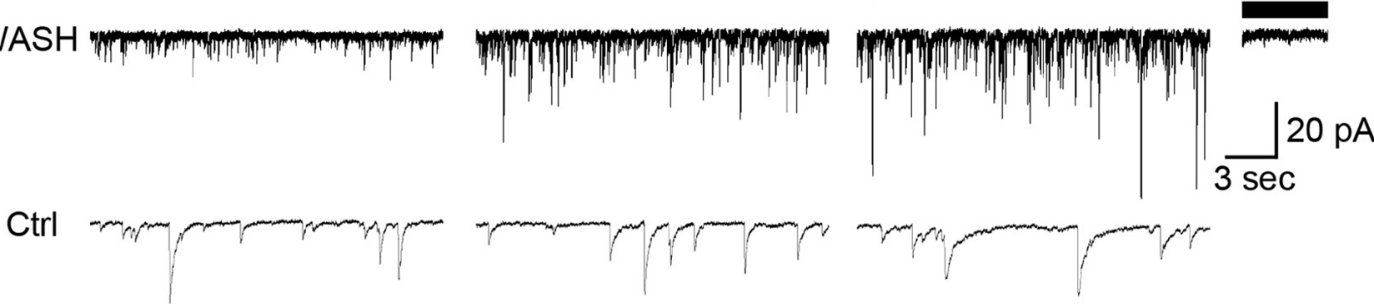

WASH
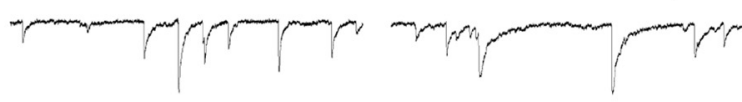

$\underset{3 \mathrm{sec}}{20 \mathrm{pA}}$

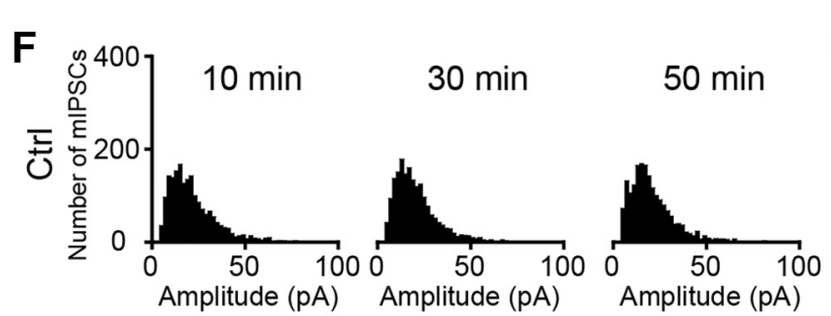

G
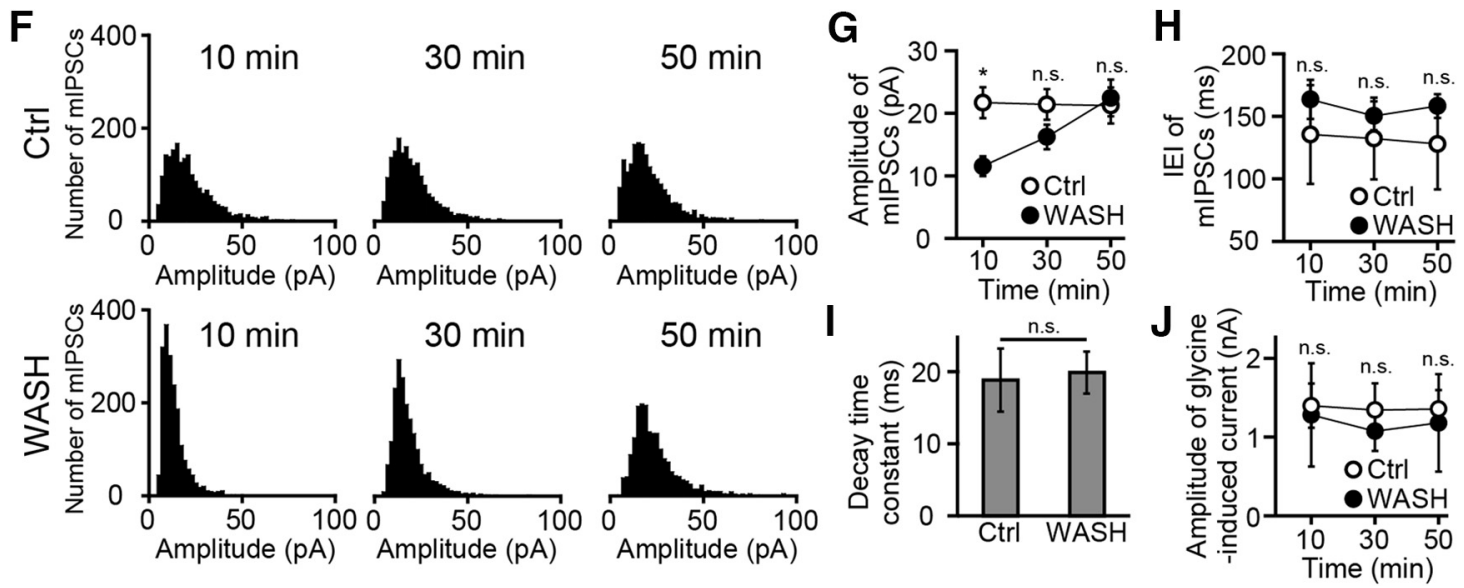

Figure 1. Activation-dependent increases in the amplitude of postsynaptic glycine responses. $\boldsymbol{A}$, Schematics of the culture model 


\section{continued}

which allows postsynaptic GlyR activation specifically after the D-H shift of the glycinergic response in mature neurons. Gly, glycine; Depol, depolarization. $\boldsymbol{B}$, Schematic timelines of the experimental protocol for patch-clamp recordings from Ctrl and strychninetreated neurons $(1 \mu \mathrm{M})$. Culture medium chronically containing STR from the 0 DIV was exchanged with a recording solution lacking strychnine (WASH). A Ctrl group acutely applied STR for $30 \mathrm{~s}$, and the solution was exchanged with a recording solution lacking strychnine. Patch-clamp recordings were started within $10 \mathrm{~min}$ following the washout of strychnine and continued for $>50$ min after strychnine removal. $\boldsymbol{A} \boldsymbol{a}-\boldsymbol{c}$ indicate the putative developmental stages shown in $\boldsymbol{A}$. $\boldsymbol{C}$, Glycine-induced currents recorded by the gramicidin-perforated patch clamp in Ctrl (left) and in cSTR (right) neurons at a holding potential of $-53 \mathrm{mV}$. Voltage ramp pulses from -113 to $+37 \mathrm{mV}$ were applied before (arrowheads, $\boldsymbol{a}$ and $\boldsymbol{c}$ ) and during (arrowheads $\boldsymbol{b}$ and $\boldsymbol{d}) 100 \mu \mathrm{m}$ glycine application. $\boldsymbol{D}$, Average $I-V$ relationship for glycinergic responses in Ctrl group ( $n=5$ cells) and cSTR group ( $n=6$ cells), as used to derive $E_{\text {gly. }}$ Superimposed histogram shows the mean values of $E_{\text {gly }} \pm$ SEM. $\boldsymbol{E}$, Representative traces of spontaneous glycinergic mIPSC at three different time points in the Ctrl group (top) and WASH group (bottom). After each recording, strychnine was applied to confirm the strychninesensitive glycinergic responses. Representative traces in $\boldsymbol{a}$ and $\boldsymbol{b}$ show longer ranges and wide-stretched views from same neurons. $\boldsymbol{F}$, The amplitude distribution histograms of glycinergic mIPSCs at three different time points (bin width, 2 pA). Each graph is constructed from pooled data of 5 min duration in the Ctrl condition ( $n=8$ cells) and the WASH condition ( $n=7$ cells). $\mathbf{G}, \boldsymbol{H}$, Time courses of mean amplitudes $(\boldsymbol{G})$ and interevent intervals $(\boldsymbol{H})$ of the glycinergic mIPSC in the Ctrl condition (open circles, $n=8$ cells) and the WASH condition (filled circles, $n=7$ cells). Each point and bar represents the mean and \pm SEM, respectively. $I$, Mean values \pm SEM of the decay time constant of the glycinergic mIPSC in the Ctrl group ( $n=8$ cells) and the WASH group ( $n=7$ cells) at 10 min after strychnine removal. $\boldsymbol{J}$, Time courses of mean amplitudes of the bath-applied $100 \mu \mathrm{M}$ glycine-induced IPSC in the Ctrl condition (open circle, $n=7$ cells) and the WASH condition (filled circles, $n=6$ cells). Glycine is rapidly applied and removed in the whole-cell body by Y-tube. Each point and bar represents the mean and \pm SEM, respectively. The significance of the difference was tested by the Student's unpaired two-tailed $t$ test $(\boldsymbol{D}, \boldsymbol{I})$ and by the Kruskal-Wallis test followed by post hoc comparisons using the Mann-Whitney $U$ test with Bonferroni's correction between Ctrl and WASH conditions at each time point $(\boldsymbol{G}, \boldsymbol{H}$, and $\boldsymbol{J})$. *p $<0.05$ (D and I) or 0.05/3 after Bonferroni's correction (G-I). n.s., Not significant.

solution to isolate GlyR responses. In the WASH group, neurons cultured in the chronic presence of strychnine, glycinergic mIPSCs were recorded $>60$ min after strychnine removal. In the Ctrl group, cultured without strychnine, neurons were transiently incubated with strychnine for $30 \mathrm{~s}$ to examine the effect of residual strychnine, before being transferred to the standard bath solution (Fig. 1B). The decay time constants of the mIPSCs at 10 min in the WASH group (mean, $19.9 \pm 2.9 \mathrm{~ms} ; n=7$ cells) were comparable to those in the Ctrl group (mean, $18.8 \pm$ $4.4 \mathrm{~ms} ; n=8$ cells; $p=0.92$, Student's unpaired twotailed $t$ test; Fig. 1/), suggesting that the developmental shift of GlyR subunit compositions was unaffected by chronic strychnine treatment. In the WASH group, the amplitude of glycinergic mIPSCs gradually increased after strychnine removal $(11.5 \pm 1.6,16.2 \pm 2.0$, and $22.4 \pm$ $2.9 \mathrm{pA}$, respectively, at 10,30 , and 50 min after strychnine removal; $n=7$; Fig. $1 E-G$ ). In contrast, the amplitudes of mIPSCs were stable in the Ctrl group across the same recording period $(21.7 \pm 2.4,21.4 \pm 2.5$, and $21.2 \pm 2.9$ $\mathrm{pA}$, respectively, at 10,30 , and $50 \mathrm{~min} ; n=8$; Fig. $1 E-G$ ). The amplitudes of mIPSCs in WASH groups was significantly lower than those in the Ctrl groups at $10 \mathrm{~min}(p=$ $0.0037)$ but were time-dependently increased in the Ctrl group ( $p=0.094$ at $30 \mathrm{~min} ; p=0.779$ at $50 \mathrm{~min}$ ). Hence, glycinergic mIPSCs gradually increase in amplitude following relief from chronic strychnine block, and this is not due to the slow washout of residual strychnine. The interevent intervals (IEIs) of mIPSCs were constant in both the Ctrl conditions $(136 \pm 16,132 \pm 11$, and $128 \pm 10 \mathrm{~ms}$, respectively, at 10,30 , and $50 \mathrm{~min} ; n=8$ ) and the WASH conditions (164 $\pm 39,150 \pm 33$, and $158 \pm 36 \mathrm{~ms}$, respectively, at 10,30 , and $50 \mathrm{~min} ; n=7$ ). This result may indicate that chronic strychnine treatment has little effect on presynaptic glycine release (Fig. 1E,H). However, a detailed analysis of synaptic numbers is required since the balance between presynaptic release probability and the number of synapses affects the IEI.

To distinguish whether the gradual increases in the amplitudes of mIPSCs in the WASH group is due to the increase in the number of GlyRs on the whole cell surface or specifically at synapses, $100 \mu \mathrm{M}$ glycine was locally applied using Y-tube at different time points after strychnine removal. However, the amplitudes of glycine-evoked currents were similar at different time points between the Ctrl group $(1.4 \pm 0.3,1.3 \pm 0.3$, and $1.4 \pm 0.2 \mathrm{nA}$, respectively, at 10,30 , and $50 \mathrm{~min}$ after strychnine removal; $n=7)$ and the WASH group $(1.3 \pm 0.7,1.1 \pm 0.3$, and $1.2 \pm 0.6 \mathrm{nA}$, respectively, at 10,30 , and 50 min after strychnine removal; $n=8$ ) groups, and no differences were detected between these groups $(p=0.23,0.23$, and 0.19 , respectively, at 10,30 , and $50 \mathrm{~min}$; Fig. 1J), indicating that cell surface expressions of GlyRs did not change during the recording period.

\section{Chronic blockade of glycine receptors decreases GlyR cluster size at inhibitory synapses}

The time-dependent increase in mIPSC amplitudes following receptor blockade may be associated with changes in postsynaptic receptor clusters. To examine this, we prepared three groups of cultured spinal cord neurons: cultures without strychnine (Ctrl), with chronic strychnine (cSTR), and with chronic strychnine for all but the $1 \mathrm{~h}$ prior to experiments (WASH; Fig. 2A). Localization of glycine receptors at inhibitory synapses was evaluated by double-immunostaining using antibodies against a presynaptic inhibitory terminal marker, the VIAAT (or VGAT), and against the GlyR $\alpha 1$ subunit (mAb2b), a postsynaptic glycine receptor marker (Fig. $2 B$ ). In the total numbers of VIAAT puncta (cSTR group, $833 \pm 67, n=17$, $p=1.00$ vs Ctrl group; WASH group, $798 \pm 83, p=1.00$ vs Ctrl group; Ctrl group, $739 \pm 71)$ and GlyR puncta 

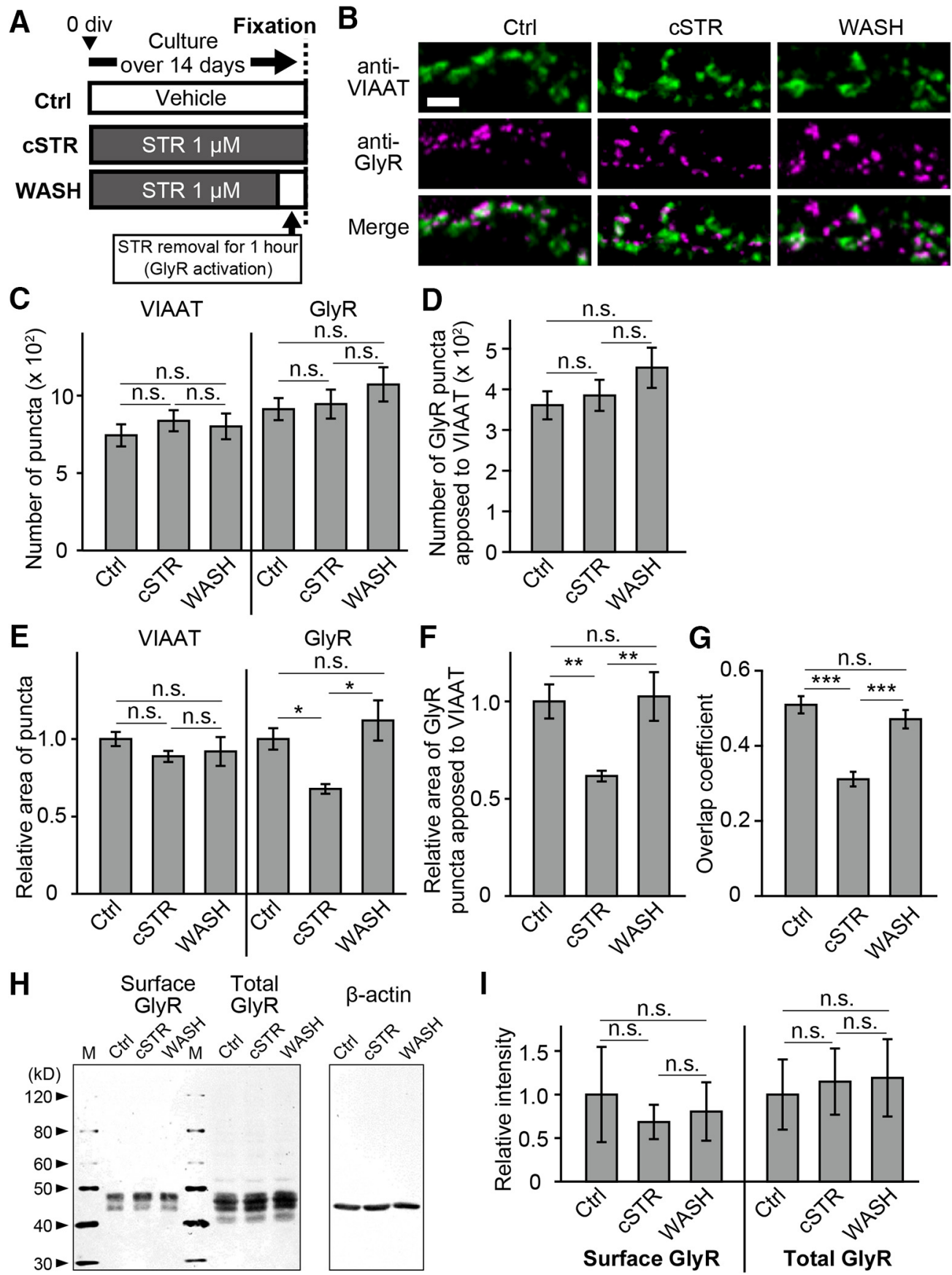

Figure 2. Strychnine removal restores synaptic GlyR clusters. A, Schematic timelines of the experimental protocol. Three experimental groups consisted of neurons incubated with only the vehicle (Ctrl group), $1 \mu \mathrm{m}$ strychnine chronically (cSTR group), or $1 \mu \mathrm{m}$ strychnine until $1 \mathrm{~h}$ before the experiment (WASH group). Neurons in all groups were subsequently fixed by $4 \%$ PFA. B, Representative double-immunostaining images of VIAAT (green) and GlyRs (magenta) in fixed cultured neurons in Ctrl, cSTR, and WASH conditions. Scale bar, $2 \mu \mathrm{m}$. $\boldsymbol{C}$, The number of total VIAAT and GlyR punctate signals per neurons in Ctrl, cSTR, and WASH conditions. $\boldsymbol{D}$, The number of GlyR punctate signals apposed to VIAAT per neurons in Ctrl, cSTR, and WASH conditions. $\boldsymbol{E}$, The relative areas of total VIAAT and GlyR punctate signals in Ctrl, cSTR, and WASH conditions. $\boldsymbol{F}$, The relative areas of GlyR signals apposed to VIAAT in Ctrl, cSTR, and WASH conditions. G, Overlap coefficients of Manders et al. (1993) for VIAAT and GlyR signals. $\boldsymbol{H}$, A representative immunoblot analysis of total and surface expression levels of glycine receptors in Ctrl, cSTR, and WASH conditions. Biotinylated glycine receptors (surface) were isolated from the detergent-soluble fraction (total). Expression levels of $\beta$-actin were used as an internal control for total GlyR. M, Molecular weight marker. I, Quantification of total and surface expression levels of glycine receptors. Expression levels of total GlyRs are normalized by $\beta$-actin, and those of surface GlyRs are normalized by total GlyRs. $\mathbf{C}-\mathbf{G}, \boldsymbol{I}$, Statistical data are reported as the mean \pm SEM derived from $14-17$ cells in three independent experiments $(\boldsymbol{C}-\mathbf{G})$ and from three independent experiments (I). The relative numbers, areas, and intensities are normalized to the mean values of Ctrl conditions. $\boldsymbol{C}-\mathbf{G}, \boldsymbol{I}$, The significance of the difference was tested by one-way ANOVA with Bonferroni's post hoc test $(\boldsymbol{C}-\mathbf{G})$ and by Kruskal-Wallis test followed by post hoc comparisons using Mann-Whitney $U$ test with Bonferroni's correction (I). *p $<0.05$, **p $<0.01$, *** $p<0.001$. n.s. Not significant. 
(cSTR group, $942 \pm 94, p=1.00$, vs Ctrl group; WASH group, $1068 \pm 110, p=0.76$ vs Ctrl group; Ctrl group, 909 \pm 72 ), no differences were detected among the three groups (Fig. 2C). Similarly, the numbers of GlyR $\alpha 1$ puncta colocalized to VIAAT puncta were not different among all three conditions (cSTR, $383 \pm 38, p=1.00$ vs Ctrl; WASH, $451 \pm 49, p=0.40$, vs Ctrl; Ctrl, $359 \pm 34$; Fig. $2 D$ ). Although no differences were observed in the relative size of VIAAT puncta across the three groups (cSTR group, $0.89 \pm 0.04, p=0.58$ vs Ctrl group; WASH group, $0.92 \pm 0.09, p=1.00$ vs Ctrl; Ctrl group, $1.00 \pm 0.05$ ), the relative size of GlyR puncta was significantly smaller in the cSTR group than those in Ctrl and WASH groups (cSTR group, $0.68 \pm 0.03, p=0.02$ vs Ctrl; WASH group, 1.12 $\pm 0.13, p=0.98$ vs Ctrl; Ctrl group, $1.00 \pm 0.07$; Fig. 2E). In addition, the relative size of GlyR $\alpha 1$ puncta apposed to VIAAT signals in the cSTR group $(0.62 \pm 0.03)$ was significantly smaller than those of GlyR $\alpha 1$ puncta in both the Ctrl group (1.00 $\pm 0.09 ; p=0.007)$ and the WASH group $(1.03 \pm 0.13 ; p=0.004 ;$ Fig. $2 F)$. In addition, the extent of colocalization of the VIAAT and GlyR $\alpha 1$ puncta was significantly lower in the cSTR group (overlap coefficient, $0.31 \pm 0.02)$ compared with that in the Ctrl group $(0.51 \pm$ $0.02 ; p<0.0001)$ and the WASH group $(0.47 \pm 0.02 ; p<$ 0.0001 ; Fig. 2G). The total number of GlyR $\alpha 1$ proteins expressed in the cells (cSTR group, $p=1.00$ vs Ctrl group; WASH group, $p=1.00$ vs Ctrl group), or the total number of GlyR $\alpha 1$ proteins expressed on the plasma membrane were not significantly different across all three experimental groups, as measured by Western blotting (cSTR group, $p=1.00$ vs Ctrl group; WASH group, $p=$ 1.00 vs Ctrl group; Fig. $2 H, I)$. This indicates that chronic treatment with strychnine reduces the size of GlyR clusters localized at inhibitory synapses, without affecting the numbers of total and synaptic GlyR clusters. The distribution of GlyR cluster size and localization returns to control levels within $1 \mathrm{~h}$ of strychnine washout. Hence, in these mature cultures, synaptic GlyR cluster size is rapidly increased following receptor activation, coincident with a change in surface GlyR localization.

In addition, chronic strychnine treatment does not affect the synaptic number (Fig. 2D). Thus, the comparable length of IEls in mIPSCs between the WASH and Ctrl groups (Fig. 1E,H) indicates that presynaptic release probability is unaffected by chronic strychnine treatment.

\section{GlyR activation reduces the spatial dynamics of laterally diffusive GlyRs in the cytoplasmic membrane}

The increase in GlyR cluster size and change in surface localization without any apparent change in total cellular or surface expression of GlyRs suggests that GlyR activation somehow changes GlyR surface distribution to recruit receptors to synaptic clusters. To examine the behavior of surface-localized GlyRs, we applied FRAP to single neuronal processes (Soumpasis, 1983; Reits and Neefjes, 2001). To selectively visualize surface GlyRs, we generated a GlyR $\alpha 1$ construct tagged with SEP to give a $\mathrm{pH}$-sensitive enhanced green fluorescent protein sequence in the extracellular N-terminal domain of GlyR $\alpha 1$ subunit (SEP-GlyR). SEP emits a distinctively high fluorescence when in a neutral $\mathrm{pH}$ environment but does not fluoresce when within a more acidic environment, such as that found inside intracellular transport organelles, and hence this enables the selective visualization of the surface population of proteins (Miesenböck et al., 1998; Sankaranarayanan and Ryan, 2001; Ashby et al., 2004). The fluorescence intensity of SEP-GlyRs on single neuronal processes in the three groups was measured before and after photobleaching (Fig. 3A). Localized photobleaching eliminates fluorescent signals from SEP-GlyRs within the targeted region, and hence recovery of the fluorescent signal reflects the movement of unbleached SEP-GlyRs into the target region, a so-called "mobile fraction," that was quantified as the relative recovery of fluorescence at the end of the $257 \mathrm{~s}$ imaging period after photobleaching (Fig. 3B,C). In neurons from the Ctrl group, the mobile fraction reached a plateau of $0.18 \pm$ $0.03(n=15)$, which was similar to that observed in the WASH group ( $0.14 \pm 0.03 ; n=12 ; p=0.52)$. In contrast, the mobile fraction in the cSTR group was significantly higher $(0.46 \pm 0.03 ; n=16 ; p<0.0001)$, indicating an increase in the population of new or mobile SEP-GlyRs that reappear at the neurite plasma membrane after photobleaching. Conversely, the lower mobile fractions in the Ctrl and WASH groups suggest that GlyRs in these groups are less mobile or that receptor activation makes them more stable.

The increase in GlyR-SEP fluorescence after photobleaching may arise from either (1) exocytosis of GlyRs from cytoplasmic vesicles into the plasma membrane or (2) lateral diffusion of surface GlyRs from outside of the bleached region. To distinguish which of these is increased by chronic strychnine administration, we measured the fluorescence recovery in chronically strychninetreated neurons in the presence of BFA, an inhibitor of ER-Golgi transport that blocks exocytosis (Fujiwara et al., 1988; Rannals and Kapur, 2011). BFA (5 $\mu \mathrm{g} / \mathrm{ml}$ ) was added to the external solution for 90 min before each imaging. The mobile fraction in the presence of BFA was also elevated relative to control and was not significantly different from that seen in the cSTR group (cSTR plus BFA: $0.54 \pm 0.03, n=5, p=0.11$; Fig. 3B,C).

We therefore next examined whether the higher mobile fraction of SEP-GlyR in the cSTR group is due to an increase in the appearance of new GlyRs from lateral diffusion, using a combination of FRAP with FLIP (Jaskolski et al., 2009; Hildick et al., 2012). FLIP involved repeated photobleaching of regions bilateral to the center region targeted by FRAP, so that GlyRs moving into the center target region are photobleached before they arrive (Fig. 3D,E). Thus, FLIP excludes laterally diffusing GlyRs from the mobile fraction, leaving just newly exocytosed SEP-GlyR $\alpha 1$ to contribute to the fluorescence recovery. The mobile fractions in these FRAP-FLIP experiments were comparable among the experimental groups (cSTR group, $0.14 \pm 0.02, n=13, p=0.70$ vs Ctrl group; WASH group, $0.11 \pm 0.02, n=11, p=0.09$ vs Ctrl group; Ctrl group, $0.17 \pm 0.03, n=8$; Fig. $3 F, G$ ). Together, these results demonstrate that chronic strychnine administration 
A

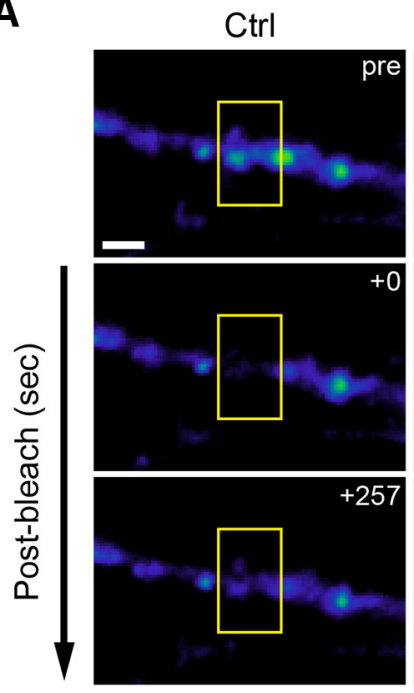

B

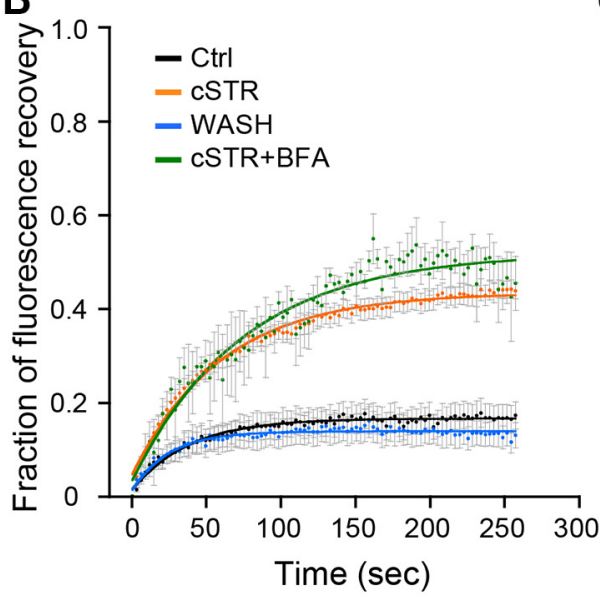

D

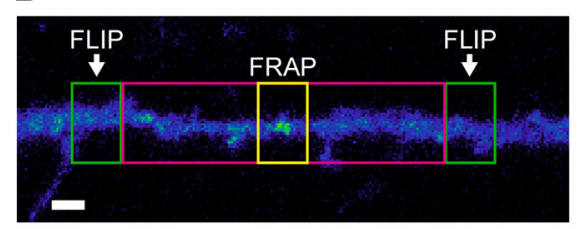

F

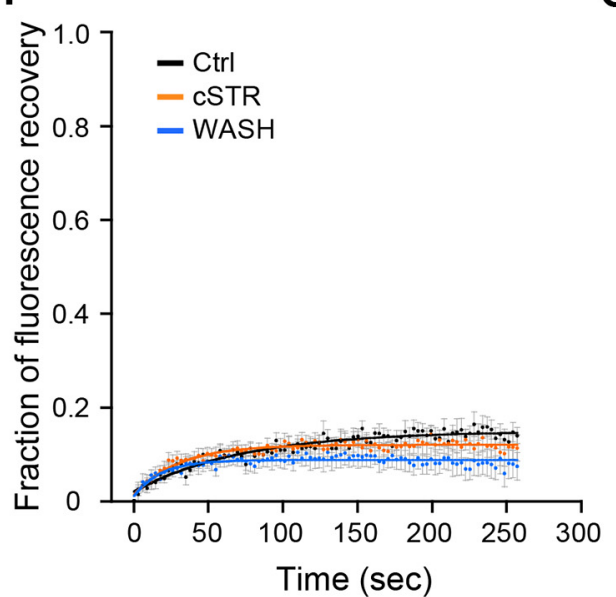

CSTR
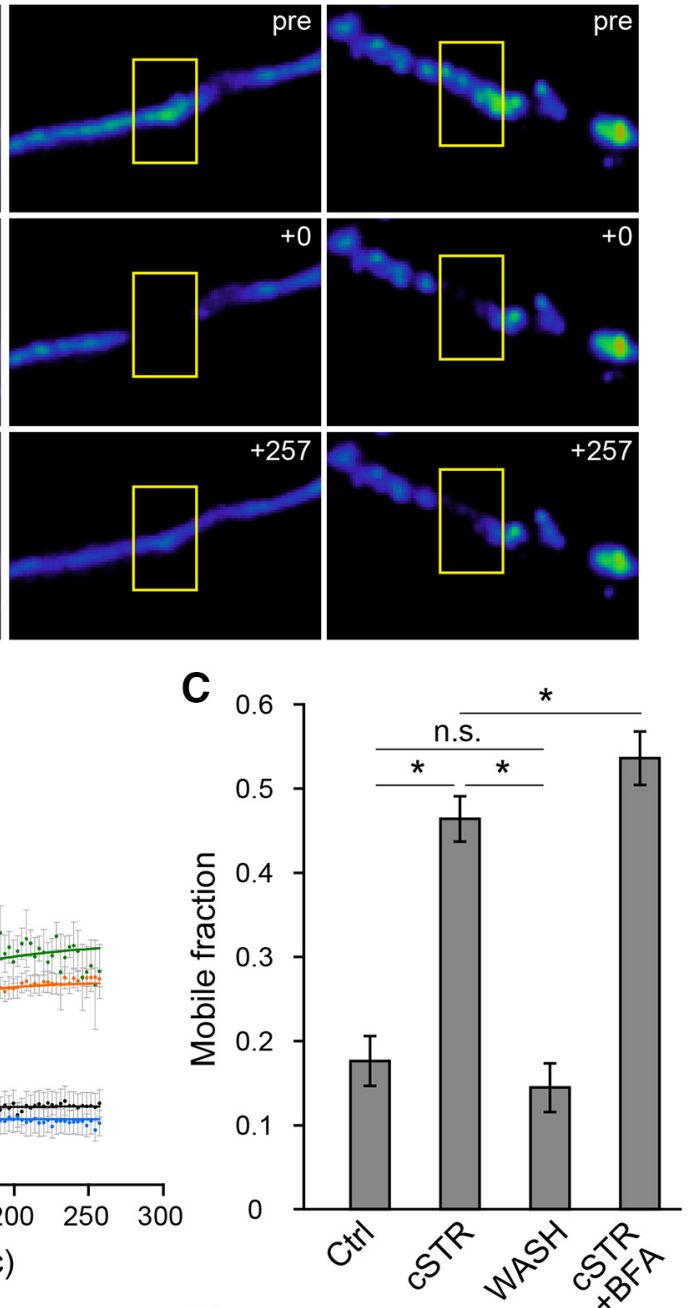

E

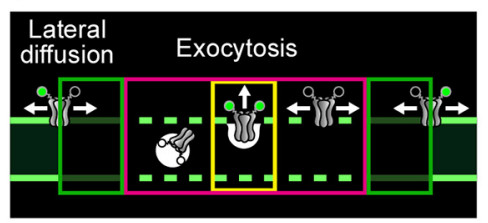

G

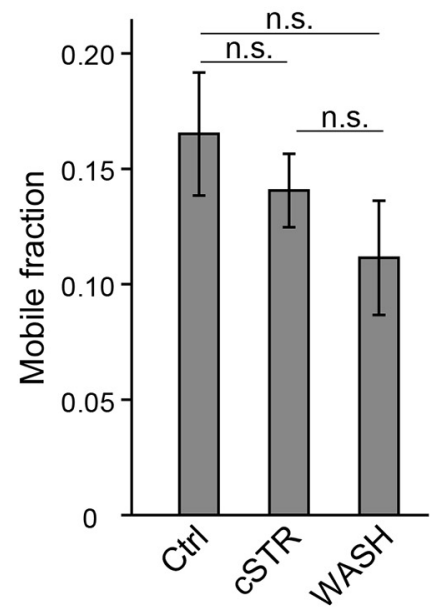

Figure 3. Cell surface dynamics of glycine receptors in live neurons. A, FRAP in neuronal processes expressing SEP-GlyR $\alpha 1$. 
continued

Fluorescence of SEP-GlyR $\alpha 1$ was bleached in the region of the neuronal process indicated by the yellow rectangles. Each row represents before (pre), immediately after $(+0 \mathrm{~s})$, and at a later time $(+257 \mathrm{~s})$ after photobleaching in each condition (Ctrl, cSTR, and WASH). Scale bar, $2 \mu \mathrm{m}$. B, Normalized fluorescence recovery curves after photobleaching in Ctrl (black, $n=15$ cells), cSTR (orange, $n=16$ cells), WASH (cyan, $n=12$ cells), and cSTR + BFA (green, $n=5$ cells) conditions. Each plot and bar represents the mean and \pm SEM. $\boldsymbol{C}$, Averaged mobile fractions in Ctrl, cSTR, WASH, and cSTR with BFA conditions (mean \pm SEM), as shown in $\boldsymbol{B}$. The mobile fraction was defined as the extent of fluorescence recovery at the end of the imaging time. $\boldsymbol{D}, \mathrm{A}$ representative image of SEP-GlyR $\alpha 1$ fluorescence and the regions of the neuronal processes used for the FRAP-FLIP experiments. Repetitive photobleaching (FLIP, green rectangles) occurred at regions bilateral to the central FRAP region (yellow rectangle). A magenta rectangle shows the buffer region used to minimize any effects of the leakage of light from the FLIP regions. Scale bar, $2 \mu \mathrm{m}$. $\boldsymbol{E}$, A schematic of hypothetical SEP-GlyR $\alpha 1$ movements under the FRAP-FLIP configuration shown in $\boldsymbol{D}$. $\boldsymbol{F}$, Normalized fluorescence recovery curves (mean \pm SEM) in Ctrl ( $n=8$ cells, black), cSTR ( $n=15$ cells, orange), and WASH ( $n=12$ cells, cyan) conditions in the FRAP-FLIP experiments. G, Averaged mobile fractions in the Ctrl, cSTR, and WASH conditions (mean \pm SEM), as shown in $\boldsymbol{F}$. The significance of difference was tested by Kruskal-Wallis test followed by post hoc comparisons using the Mann-Whitney $U$ test with Bonferroni's correction. $* * * p$ $<0.001 / 6$ after Bonferroni's correction for the six tests. n.s., Not significant.

does not affect the rate of exocytosis of new GlyRs from intracellular stores into the plasma membrane but, rather, increases the lateral diffusion of GlyRs along the plasma membrane.

\section{GlyR dynamics at gephyrin-expressing postsynapses}

Changes of the diffusion properties of receptors can determine the receptor localization at synapses (Dahan et al., 2003; Bannai et al., 2009; Choquet and Triller, 2013). The postsynaptic localization and cluster sizes of GlyRs are regulated by gephyrin, a major scaffolding protein of GlyRs (Kirsch and Betz, 1995; Calamai et al., 2009). The distribution of gephyrin has been reported to be either affected (Kirsch and Betz, 1998) or unaffected (Lévi et al., 1998; Tyagarajan and Fritschy, 2014) by GlyR activation. Hence, we examined whether chronic strychnine treatment affects the distribution of gephyrin at inhibitory synapses in the present study by measuring the overlap coefficient of Manders et al. (1993) between immunostained gephyrin and VIAAT puncta. Neither chronic strychnine application (cSTR group) nor the wash from chronic strychnine with subsequent GlyR activation (WASH group) affected the gephyrin distribution (cSTR group, $0.53 \pm 0.01, p=1, n=15$ vs Ctrl group; WASH group, $0.52 \pm 0.01, p=0.57, n=16$, vs Ctrl group; Ctrl group, $0.54 \pm 0.01, n=13$; Fig. $4 A, B$ ).

Next, we examined whether the GlyR activationinduced change in GlyR mobility was selective for regions around inhibitory postsynapses, as defined by gephyrinpositive locations. Neuronal cultures were cotransfected with both SEP-GlyR $\alpha 1$ and mCherry-GPHN, and the fluorescence intensity of SEP-GlyR $\alpha 1$ was measured before and after photobleaching and was compared between mCherry-GPHN-positive and mCherry-GPHN-negative regions (Fig. 4C). At mCherry-GPHN-positive spots, the mobile fraction of SEP-GlyR $\alpha 1$ was larger in the chronic presence of strychnine (cSTR; $0.48 \pm 0.06, n=8, p=$ 0.002 ) in comparison with the mobile fraction of SEPGlyR $\alpha 1$ s at mCherry-GPHN-positive spots in Ctrl cultures $(0.23 \pm 0.03, n=14)$. Although ectopic expression of SEP-GlyR $\alpha 1$ s are likely to form homomeric GlyRs, synaptic localization of GlyRs is regulated by interactions between gephyrin and heteromeric GlyRs containing $\alpha 1$ and $\beta$-subunits (Tyagarajan and Fritschy, 2014). Thus, we also measured the mobile fraction of SEP-GlyR $\alpha 1$ in the chronic presence of ginkgolide B (cGinkB), a potent channel blocker of heteromeric GlyR (Kondratskaya et al., 2005; $0.40 \pm 0.06, n=8$ ) and confirmed that it was significantly larger than that in the Ctrl group $(p=0.005)$. In contrast, the mobile fraction in the WASH group $(0.25 \pm$ $0.03, n=15)$ was similar to that in the Ctrl group ( $p=$ 0.62; Fig. $4 D, E$ ). However, the mobile fractions at $\mathrm{mCherry-GPHN-lacking} \mathrm{regions} \mathrm{were} \mathrm{all} \mathrm{relatively} \mathrm{high,}$ with no significant differences among the groups (Ctrl group, $0.47 \pm 0.04, n=14$; cSTR group, $0.59 \pm 0.07, n$ = 8; cGinkB group, $0.62 \pm 0.04, n=8$; WASH group, 0.53 $\pm 0.05, n=15$; Fig. 4F,G). Although high-power excitation laser light also decreased the fluorescence intensity of mCherry-GPHN, the mobile fractions of fluorescence recovery between SEP-GlyR $\alpha 1$ and mCherry-GPHN in the same FRAP regions in the sum of the three experimental groups were not correlated $(r=0.20, p=0.25, n=37$; Fig. $4 H$ ). Thus, mobile fractions of SEP-GlyR $\alpha 1$ were independent of the photobleaching of mCherry-GPHN. These results suggest that the lateral mobility of GlyRs on the cell surface under intact glycinergic transmission is lower at sites of gephyrin localization, and this receptor stabilization is absent in chronically inactivated GlyRs. In addition, $1 \mu \mathrm{M}$ strychnine is reported to affect nicotinic acetylcholine receptors (nAChRs; Matsubayashi et al., 1998). However, the mobile fraction of SEP-GlyR $\alpha 1$ in the chronic presence $0.5 \mu \mathrm{M}$ mecamylamine hydrochloride (cMec), a noncompetitive antagonist of nAChRs (Papke et al., 2001; Yasuyoshi et al., 2002; cMec, $0.28 \pm 0.06, n$ $=8)$ was comparable to that in the Ctrl group $(p=0.62)$ in mCherry-GPHN-positive regions (Fig. 4E). Thus, higher GlyR mobility in the cSTR group is independent of $n A C h R$ blockade.

We next confirmed whether the local glycine application directly and selectively induces GlyR clustering at the gephyrin-positive area. The $1 \mathrm{~m}$ glycine was applied using a micropipette placed beside single dendrites in cSTR group. After glycine application for $60 \mathrm{~min}$, the fluorescence intensity of SEP-GlyR $\alpha 1$ was increased at gephyrin-positive spots, and the changes are significantly larger in the $\mathrm{GPHN}^{+}$area $(1.49 \pm 0.15, n=7$ cells) than in the $\mathrm{GPHN}^{-}$area $(0.95 \pm 0.06, n=7$ cells; $p=0.01$; Fig. $4 /, J)$. These results suggest that activated GlyRs make clusters selectively at gephyrin-expressing postsynapses within $60 \mathrm{~min}$. 
A

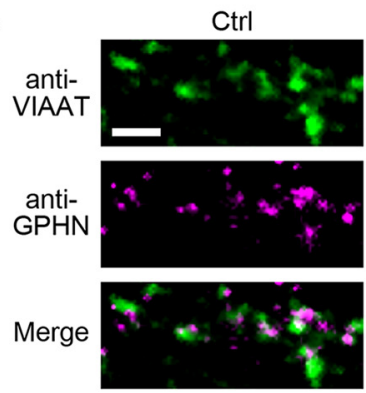

C
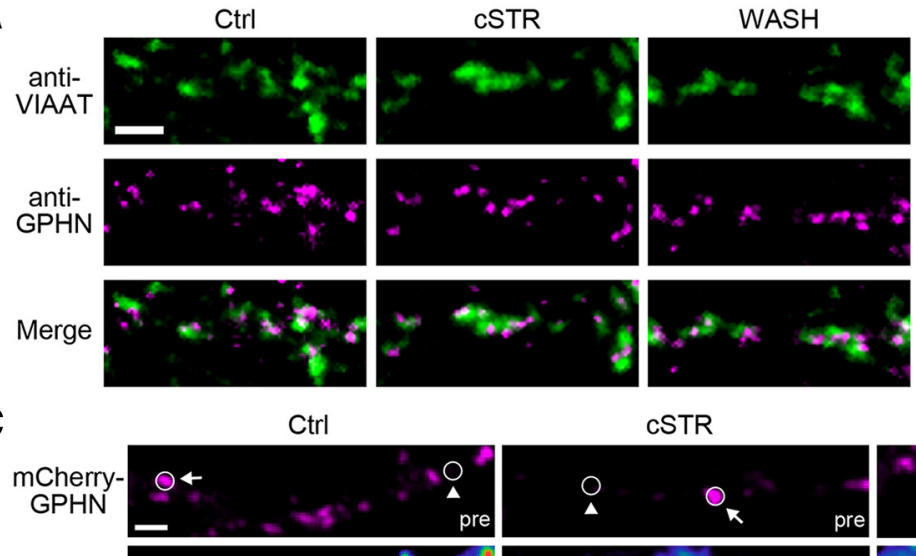

cSTR
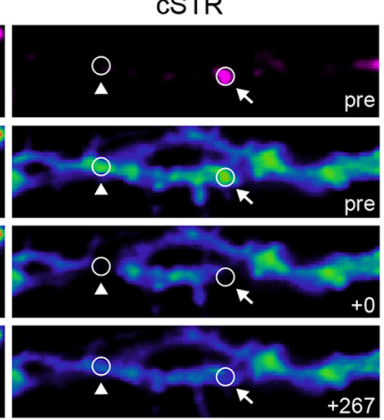

D

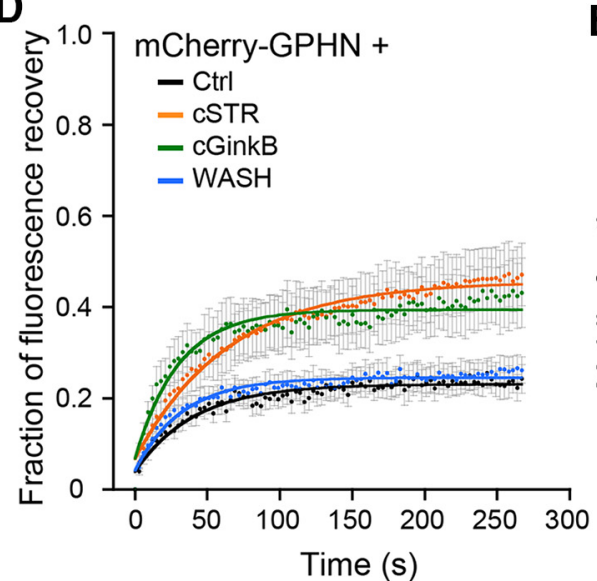

F

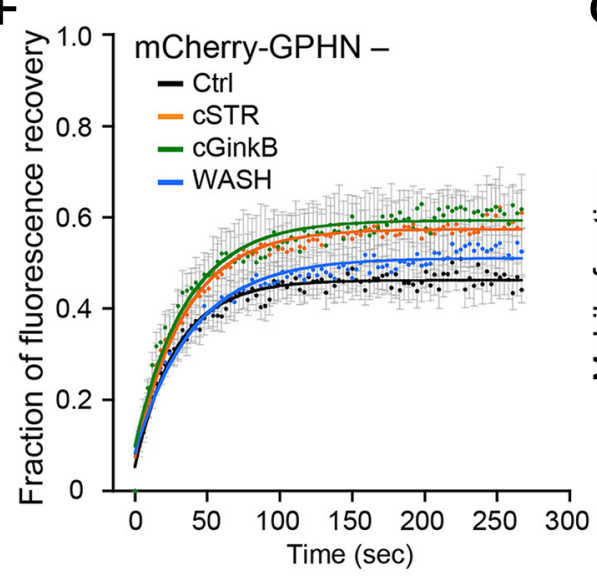

E

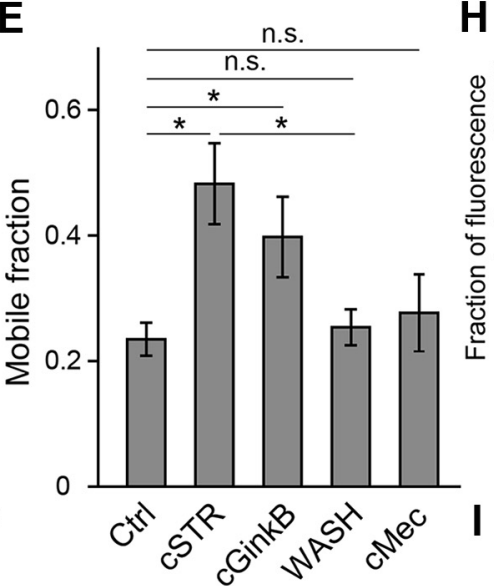

G

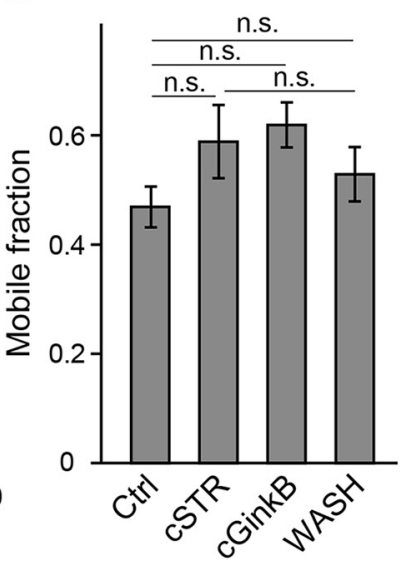

H
B

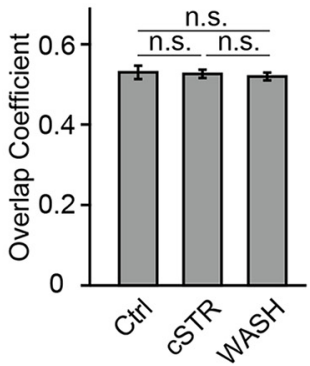

WASH
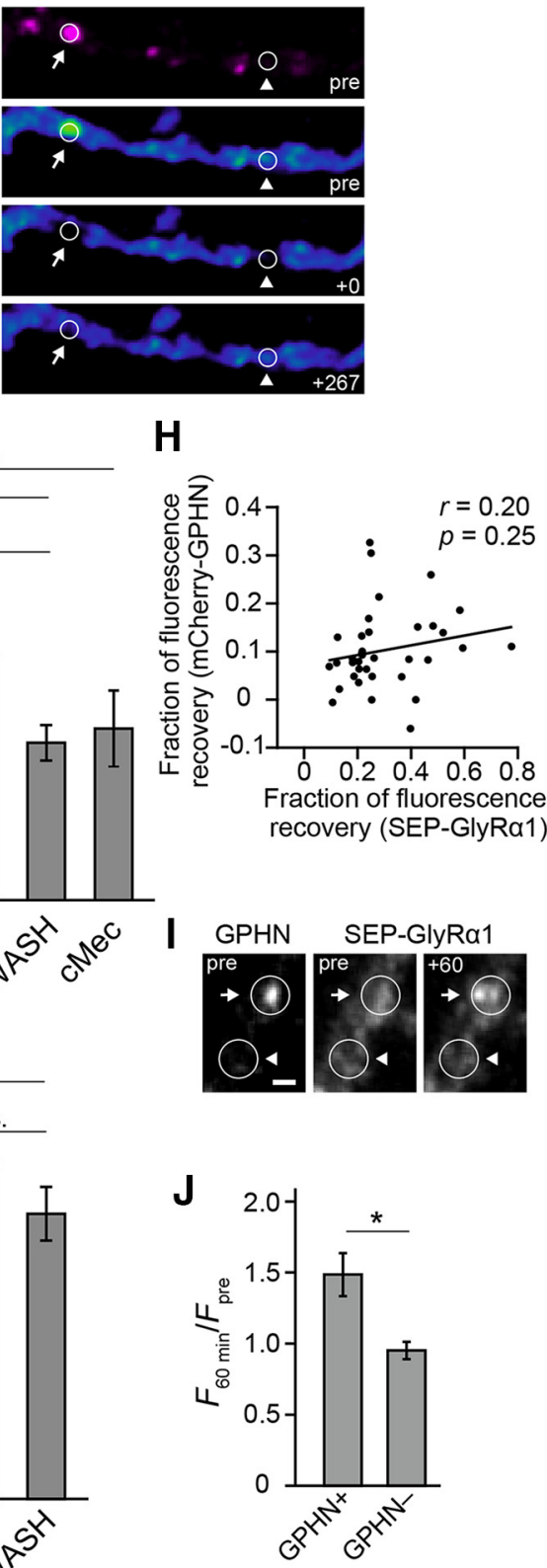

Figure 4. Glycine receptor dynamics at gephyrin-identified synaptic zones. $\boldsymbol{A}$, Representative double-immunostaining images of VIAAT (green) and GPHN (red) in fixed cultured neurons in Ctrl, cSTR, and WASH conditions. Scale bar, $2 \mu \mathrm{m}$. B, Overlap coefficients of Manders et al. (1993) of VIAAT and GPHN signals ( $n=13-16$ cells). C, Representative images of neuronal processes coexpressing SEP-GlyR $\alpha 1$ and mCherry-GPHN in Ctrl, cSTR, and WASH conditions. Top, Expression of mCherry-GPHN fluorescence. FRAP of SEP-GlyR $\alpha 1$ was measured at focal spots with (arrows) and without (arrow heads) mCherry-GPHN signals. Each row represents before (pre), immediately after $(+0 \mathrm{~s})$, and at a later time $(+267 \mathrm{~s})$ after photobleaching. Scale bar, $2 \mu \mathrm{m}$. $\boldsymbol{D}$, Fluorescence recovery 


\section{continued}

curves after photobleaching at focal spots expressing mCherry-GPHN in control (black), cSTR (orange), cGinkB (green), and WASH (cyan) conditions. Each point and bar represents the mean and \pm SEM, respectively. $E$, Averaged mobile fractions at focal spots expressing mCherry-GPHN in Ctrl ( $n=14$ cells), cSTR ( $n=8$ cells), cGinkB ( $n=8$ cells), WASH $(n=15$ cells), and cMEC ( $n=8$ cells) conditions (mean \pm SEM) for the data shown in $\boldsymbol{D}$. $\boldsymbol{F}$, Fluorescence recovery curves after photobleaching at $\mathrm{mCherry}^{-G P H N}{ }^{-}$regions in control (black), cSTR (orange), cGinkB (green), and WASH (cyan) conditions. Each point and bar represents mean and \pm SEM, respectively. G, Average mobile fraction at focal spots lacking mCherry-GPHN in Ctrl ( $n=14$ cells), cSTR $(n=8$ cells), cGinkB ( $n=$ 8 cells), and WASH $(n=15$ cells) conditions (mean \pm SEM), for the data shown in $\boldsymbol{F}$. $\boldsymbol{H}$, The correlation between fractions of fluorescence recovery of SEP-GlyR $\alpha 1$ and mCherry-GPHN ( $n=37$ cells). Each plot represents individual samples in Ctrl, cSTR, and WASH groups. I, Representative fluorescence images of mCherry-GPHN and SEP-GlyR $\alpha 1$ at a single dendrite before (pre) and after (+60) local application of glycine for $60 \mathrm{~min}$. Arrows and arrowheads shows $\mathrm{GPHN}^{+}$and GPHN ${ }^{-}$zones. Scale bar, $1 \mu \mathrm{m}$. J, Average changes of SEP-GlyR $\alpha 1$ fluorescence intensity in the $\mathrm{GPHN}^{+}$and $\mathrm{GPHN}^{-}$zones at single dendrite $(n=7$ cells). The significance of the difference was tested by one-way ANOVA with Bonferroni's post hoc test (B), by Kruskal-Wallis test followed by post hoc comparisons using the Mann-Whitney $U$ test with Bonferroni's correction $(\boldsymbol{E}, \boldsymbol{G})$ and Student's paired $(\boldsymbol{H})$ or unpaired $(\boldsymbol{J})$ two-tailed $t$ test. The Pearson's correlation coefficients $(r)$ and $p$ values $(p)$ are indicated in $\boldsymbol{H}$. $* p<0.05 / 10$ after Bonferroni's correction for the 10 tests $(\boldsymbol{E})$ and $p<0.05(\boldsymbol{J})$. n.s., Not significant.

\section{Glycinergic transmission regulates the formation of synaptic GlyR clusters, but not their maintenance}

The results above indicate that GlyR activation is required for the formation of large, stable GlyR clusters at gephyrin-positive synapses, but it is not clear whether stabilized GlyRs remain synapses only during the period they are activated or are stabilized for a longer duration without further activation. Then, after $14 \mathrm{~d}$ of neuronal culture in the absence of any GlyR block, we applied $1 \mu \mathrm{M}$ strychnine for $48,36,24$, and $1 \mathrm{~h}$ or just immediately $(0 \mathrm{~h})$ prior to acquiring imaging data (Fig. 5A). FRAP was used to measure SEP-GlyR $\alpha 1$ s fluorescence intensity at mCherry-GPHN ${ }^{+}$spots. The results showed no significant differences were found in the mobile fractions between the groups treated $36 \mathrm{~h}$ or shorter duration as compared to control (STR0h, $0.23 \pm 0.06, n=6, p=0.78$; STR1h, $0.19 \pm 0.02, n=9, p=0.31$; STR24h, $0.32 \pm 0.04, n=$ $15, p=0.07$, STR36h, $0.39 \pm 0.04, n=11, p=0.02$, compared to Ctrl, $0.23 \pm 0.03, n=14$; Fig. $5 B, C)$. In contrast, the mobile fraction of strychnine treatment for 48 h $(0.47 \pm 0.04, n=10)$ significantly increased in comparison to control $(p=0.0001)$. Hence, the block of previously activated GlyRs for up to $24 \mathrm{~h}$ has little effect, but for $48 \mathrm{~h}$ the block increases GlyR mobility at synapses.

\section{$\mathrm{Ca}^{2+}$ and CaMKII-independent clustering of synaptic GlyRs}

Previous reports have demonstrated that synaptic GlyR accumulation in developing neurons requires $\mathrm{Ca}^{2+}$ influx and CaMKII activity (Kirsch and Betz, 1998; Lévi et al., 2008; Yamanaka et al., 2013). Thus, we also examined these in mature neurons, directly measuring whether the activation-induced GlyR stabilization was affected by a cell-permeable $\mathrm{Ca}^{2+}$ chelator (BAPTA-AM), and a CaMKII inhibitor (KN62), applying these treatments during the $1 \mathrm{~h}$ washout of chronic strychnine treatment. The mobile fraction after incubation with either BAPTA-AM $(0.20 \pm 0.03$, $n=5, p=0.50)$ or KN62 $(0.16 \pm 0.05, n=6, p=0.05)$ was not significantly different from that in the WASH group $(0.25 \pm 0.03, n=15$; Fig. $5 D, E)$, indicating that intracellular $\mathrm{Ca}^{2+}$ or CaMKII is not a prerequisite for the GlyR activation-induced decrease in lateral mobility. We further tested the involvement of protein kinase $A(P K A)$ or protein kinase $C(P K C)$ on the activation-induced GlyR clustering. The effect of phosphothioate-cAMP (RpcAMP), a cell-permeable cAMP analog antagonizing PKA activity, and GFX, a cell-permeable PKC inhibitor, are tested by application during the $1 \mathrm{~h}$ GlyR activation after the washout of chronic strychnine. The administration of Rp-cAMP showed no difference $(0.13 \pm 0.14, n=5, p=$ $0.02)$, but administration of GFX $(0.43 \pm 0.03, n=11, p=$ 0.0008 ) induced a significantly higher mobile fraction compared with that in the WASH group $(0.25 \pm 0.03, n=$ 15; Fig. 5D,E).

\section{Single-particle tracking of endogenous GlyRs at synapses}

The results above suggest that the mobility of synaptic GlyRs is dynamically modulated by its activation. To more directly measure the lateral diffusion of GlyRs at synapses, we labeled the $\alpha 1$-GlyR subunit with fluorescent QDs (Dahan et al., 2003; Lévi et al., 2008). Active synaptic regions were identified by FM4-64, a lipophilic styryl dye that gets incorporated into synaptic vesicles via endocytosis (Gaffield and Betz, 2006). Individual QD-GlyRs showed quite variable trajectories and rates of diffusion in each experimental group, although in the Ctrl and WASH groups trajectories were largely restricted to the areas around active synaptic zones (Fig. 6A). The median QDGlyR diffusion coefficients in the Ctrl group $\left(6.95 \times 10^{-3}\right.$ $\mu \mathrm{m}^{2} \mathrm{~s}^{-1}, n=101$ particles) and the WASH group $(6.39 \times$ $10^{-3} \mu \mathrm{m}^{2} \mathrm{~s}^{-1}, n=54$ particles) were similar $(p=0.79)$ but significantly lower than the median diffusion coefficient of GlyRs in the cSTR group $\left(2.46 \times 10^{-2} \mu \mathrm{m}^{2} \mathrm{~s}^{-1}, n=125\right.$ particles; Ctrl vs cSTR, $p<0.0001$; WASH vs CSTR, $p<$ 0.0001 ; Fig. $6 B, C)$. These results suggest that inactive GlyRs are highly diffusive compared with activated GlyRs, with diffusion rates returning to control values within $1 \mathrm{~h}$ of receptor activation.

To determine the effects of receptor activation on the mobility of QD-GlyRs localized at synapses, we quantified the synaptic dwell time and the confinement size of each QD-GlyR in synaptic domains. Dwell time was calculated as the longest sum of consecutive subtrajectories in which each QD-GlyR stayed within the active synaptic zone (defined as the overlap with FM4-64 fluorescence; see Materials and Methods). The average dwell time in cSTR group (2.7 $\pm 0.2 \mathrm{~s}, n=314$ particles) was shorter 
A

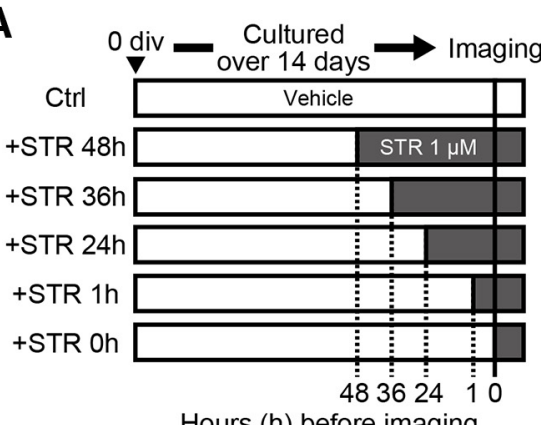

B

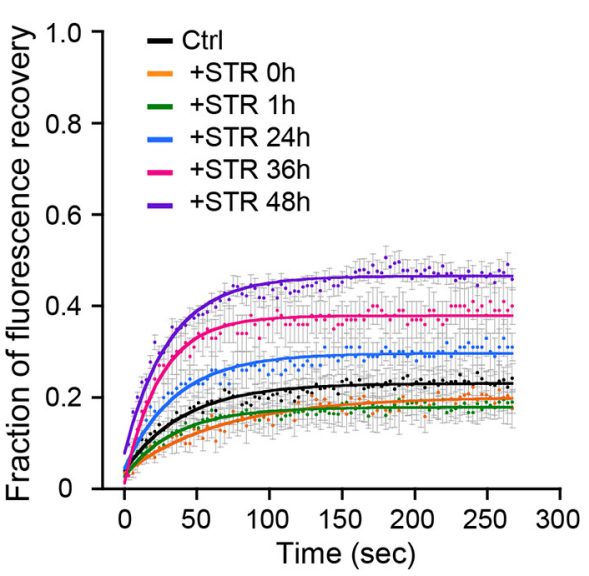

D

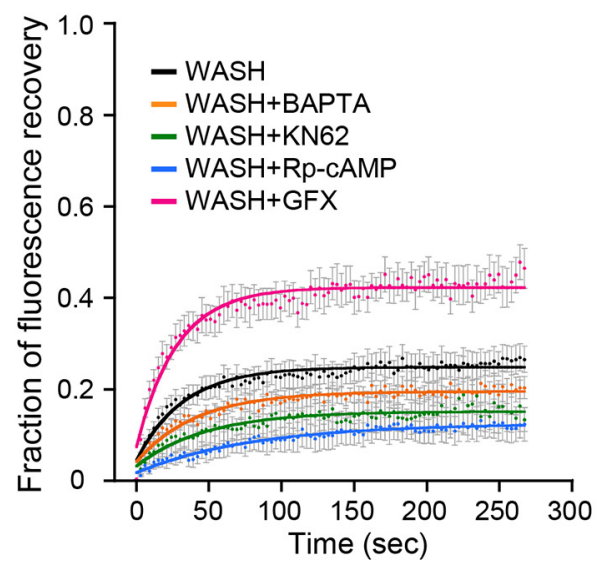

C

E

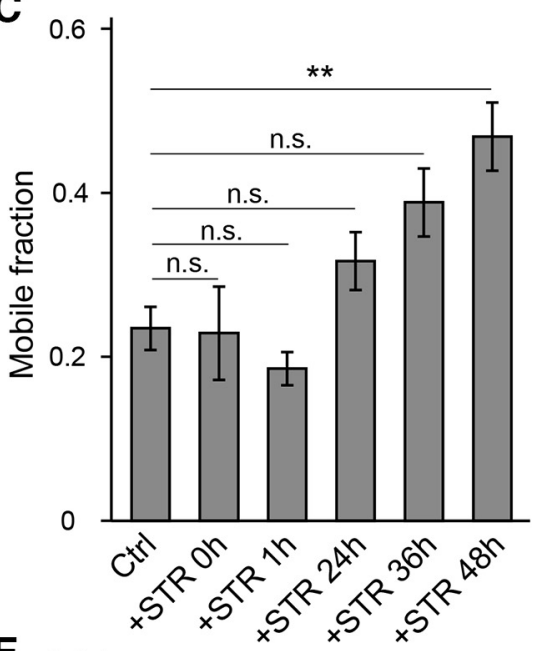

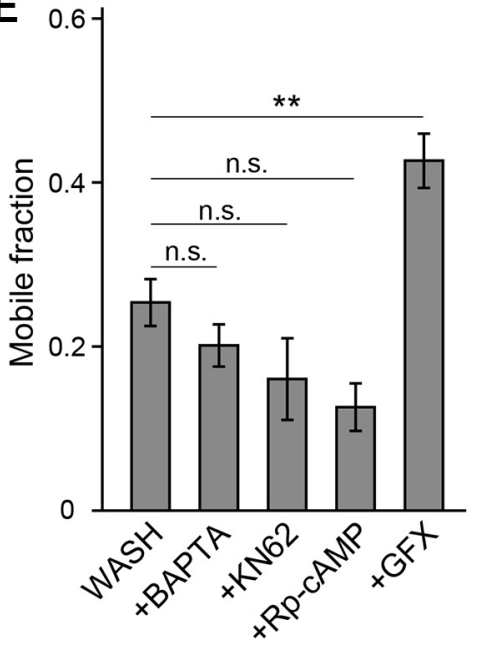

Figure 5. GlyR activation regulates the formation, but not the maintenance, of GlyR clustering. $\boldsymbol{A}$, Schematic timelines of strychnine treatments on mature neurons. Neurons cultured over 14 DIV without previous application of strychnine were applied for the administration of $1 \mu \mathrm{m}$ strychnine for $48 \mathrm{~h}$ (+STR 48h), $36 \mathrm{~h}$ (+STR 36h), $24 \mathrm{~h}(+$ STR $24 \mathrm{~h}), 1 \mathrm{~h}(+$ STR $1 \mathrm{~h})$, or immediate (+STR 0 h) before and during imaging. $\boldsymbol{B}$, Normalized fluorescence curves at focal spots with mCherry-GPHN signals in Ctrl ( $n=14$ cells, black), Ctrl with acute (+STR 0h; $n=6$ cells, orange), with $1 \mathrm{~h}$ (+STR $1 \mathrm{~h} ; n=9$ cells, green), with $24 \mathrm{~h}(+\mathrm{STR} 24 \mathrm{~h} ; n=15 \mathrm{cells}$, cyan), with $36 \mathrm{~h}$ (+STR 36h; $n=11$ cells, magenta), and with $48 \mathrm{~h}$ (+STR 48h; $n=10$ cells, purple) of STR treatment before each experiment. Each point and bar represents mean and \pm SEM, respectively. $\boldsymbol{C}$, Average mobile fractions (mean \pm SEM) shown in $\boldsymbol{B}$. $\boldsymbol{D}$, Fluorescence recovery curves after photobleaching at focal spots expressing mCherry-GPHN in WASH $(n=15$ cells, black, shown in Fig. 4D), WASH with BAPTA-AM ( $n=5$ cells, orange), WASH with KN62 ( $n=6$ cells, green), WASH with Rp-cAMP ( $n=5$ cells, cyan), and WASH with GFX ( $n=11$ cells, magenta) conditions. Each point and bar represents mean and \pm SEM, respectively. $E$, Averaged mobile fractions at focal spots expressing mCherry-GPHN in WASH ( $n=15$ cells, shown in Fig. 4E), WASH with BAPTA-AM ( $n=5$ cells), WASH with KN62 ( $n=6$ cells), WASH with Rp-cAMP ( $n=5$ cells), and WASH with GFX ( $n=11$ cells) conditions (mean \pm SEM), for the data shown in $\boldsymbol{D}$. The significance of difference was tested by the Kruskal-Wallis test followed by post hoc comparisons using the Mann-Whitney $U$ test with Bonferroni's correction. $* * p<0.01 / 15$ and $p<0.01 / 10$ after Bonferroni's correction for the 15 and 10 tests, respectively. n.s., Not significant. 
A

Ctrl

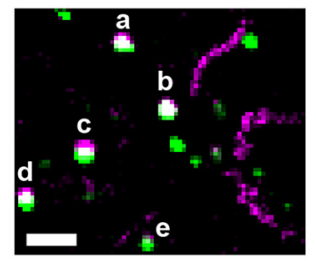

a

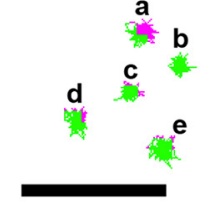

CSTR
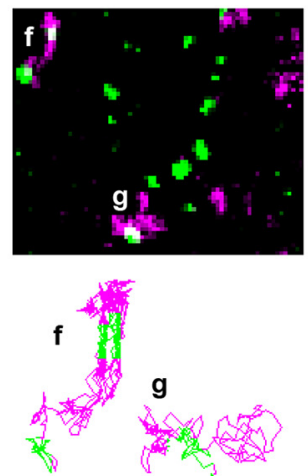

g

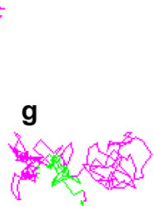

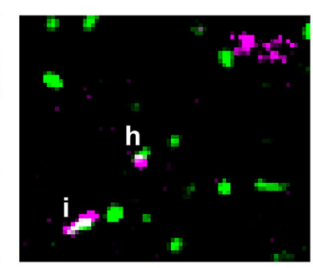

B

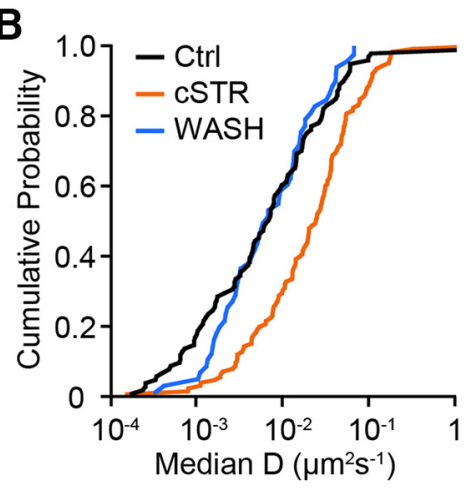

C
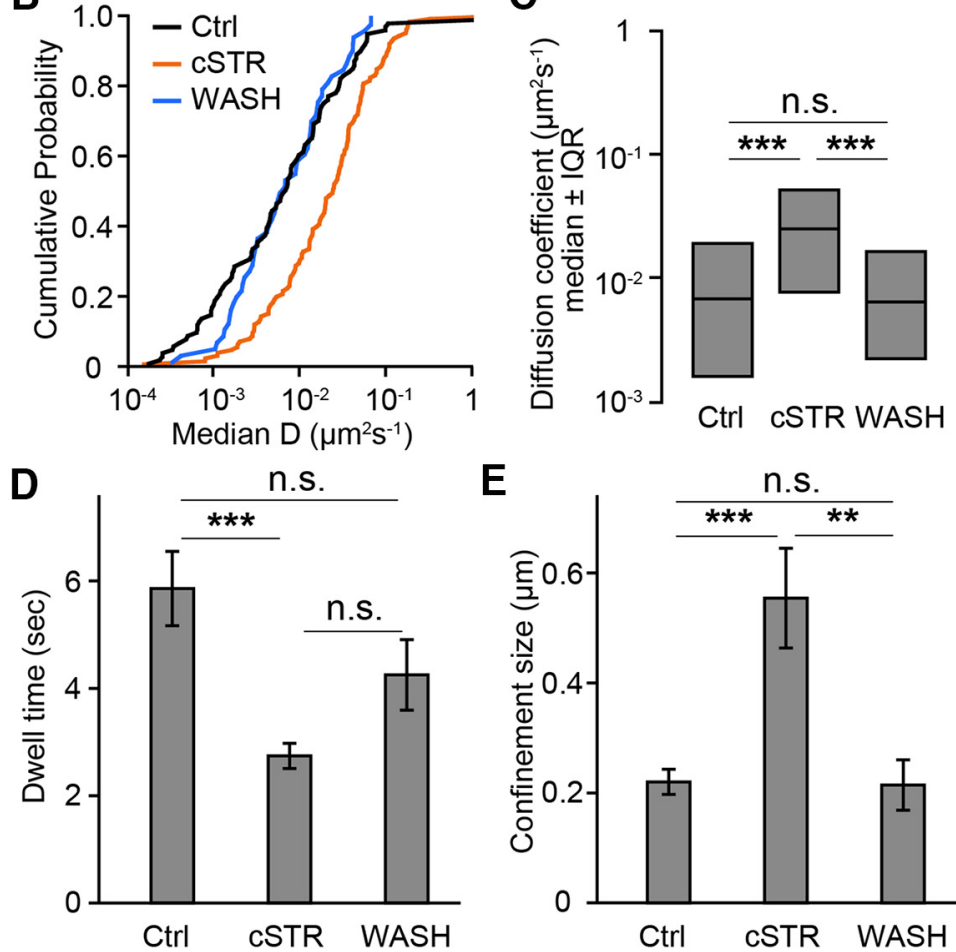

E

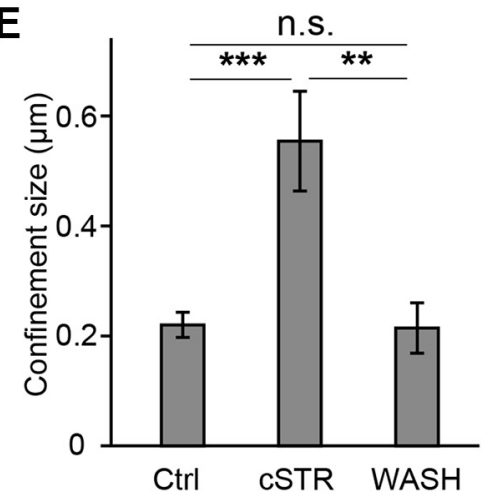

Figure 6. GlyR activation modulates its diffusion properties around active synapses. A, Representative images of QD-labeled endogenous GlyRs (magenta) and FM4-64-labeled active synapses (green) shown as maximum intensity projections of $30 \mathrm{~s}$ of image recordings in Ctrl, cSTR, and WASH conditions (top panels). The bottom panels show the trajectories of correspondent QD-labeled GlyRs (centroids) shown in the top panels inside (green) and outside (magenta) active synapses. Scale bars, $2 \mu \mathrm{m}$. $\boldsymbol{B}, \mathrm{Cumulative}$ probabilities of the median diffusion coefficient of each QD-GlyR within the active synapse region in Ctrl ( $n=101$ particles, 7 cells), cSTR ( $n=125$ particles, 8 cells), and WASH ( $n=54$ particles, 7 cells) conditions. $\boldsymbol{C}$, Median diffusion coefficients of each QD-GlyR at synapses comparing Ctrl, cSTR, and WASH conditions (median $\pm 25-75 \%$ interquartile range (IQR)). $\boldsymbol{D}$, Average dwell times of QD-GlyRs within synapse regions (Ctrl: $n=150$ particles, 7 cells; cSTR: $n=314$ particles, 8 cells; WASH: $n=97$ particles, 7 cells, mean \pm SEM). $\boldsymbol{E}$, Average confinement size of QD-GlyRs at synapses (mean \pm SEM) in Ctrl $(n=49$ particles, 7 cells), cSTR $(n=38$ particles, 8 cells), and WASH ( $n=19$ particles, 7 cells) conditions. Significance of difference was tested by Kruskal-Wallis test followed by post hoc comparisons using the Mann-Whitney $U$ test with Bonferroni's correction, $* * * p<0.001 / 3$ after Bonferroni's correction for the three tests $(\boldsymbol{C})$ and by one-way ANOVA with Bonferroni post hoc test $(\boldsymbol{D}, \boldsymbol{E})$. $* * p<0.01$, $* * * p<0.001$. n.s., Not significant.

than that of Ctrl group $(5.9 \pm 0.7 \mathrm{~s}, n=150$ particles; $p<$ $0.0001)$. The average dwell time in the WASH group ( $4.3 \pm$ $0.7 \mathrm{~s}, n=97$ particles) returned to that seen in the Ctrl group, although it was not significantly different from that in the cSTR group $(p=0.09$; Fig. $6 D)$. The confinement size of each QD-GlyR, defined as the length of an equiv- alent square within which diffusion was restricted (see Materials and Methods), was significantly larger in the cSTR condition $(0.55 \pm 0.09 \mu \mathrm{m}, n=38$ particles $)$ compared with that in the Ctrl condition $(0.22 \pm 0.02 \mu \mathrm{m}, n=$ 45 particles; $p=0.0002)$ and the WASH condition ( $0.21 \pm$ $0.05 \mu \mathrm{m}, n=19$ particles; $p=0.004$; Fig. $6 E$ ). Hence, 
chronically blocked (inactive) GlyRs diffuse at a faster rate and over a larger area in synaptic domains compared with activated GlyRs. Inactive GlyRs also reside for briefer durations at synapses.

\section{Discussion}

The present study demonstrated here that glycinergic synaptic responses and spatial distributions of GlyRs were modulated by receptor activation via presynaptically released glycine in mature spinal cord neurons independent of the $\mathrm{Ca}^{2+}$-mediated mechanism. The clustering of other ionotropic receptors, such as AMPA receptors and $\mathrm{GABA}_{\mathrm{A}}$ receptors, are regulated by a $\mathrm{Ca}^{2+}$-dependent process in both immature and mature neurons (Borgdorff and Choquet, 2002; Petrini et al., 2014). As for $\mathrm{GABA}_{\mathrm{A}}$ receptors, for example, excitatory activity induces depolarization and increases clustering of $\mathrm{GABA}_{\mathrm{A}}$ receptors via CaMKII activity (Petrini et al., 2014). The prevailing concept for GlyR synaptic clusters that was developed from studies on immature circuits is similar to that for AMPA receptors and $\mathrm{GABA}_{A}$ receptors. Glycine receptor activation induces depolarization in immature neurons and subsequent $\mathrm{Ca}^{2+}$ influx (Kirsch and Betz, 1998), which activates $\mathrm{Ca}^{2+}$-dependent kinases to elicit increases in receptor clusters (Charrier et al., 2010; Yamanaka et al., 2013). This concept relies on the fact that glycine and $\mathrm{GABA}_{\mathrm{A}}$ receptor activation induces depolarizations in early development, as intracellular $\mathrm{Cl}^{-}$is elevated due to the absence of functional $\mathrm{K}^{+}-\mathrm{Cl}^{-}$cotransporter 2 (KCC2). Since KCC2 is expressed during development, the resultant $\mathrm{Cl}^{-}$efflux lowers intracellular $\mathrm{Cl}^{-}$and converts the GABA and glycine response to hyperpolarizing (Kakazu et al., 1999; Rivera et al., 1999). This upregulation of KCC2 and switch from depolarization to hyperpolarization occurs over the first postnatal weeks in most regions of the central nervous system (Ehrlich et al, 1999; Jean-Xavier et al., 2006). In cultured neurons of spinal cord and brainstem, the switch from GABA or glycine-induced depolarizations to hyperpolarizations occurs between 1 and 3 weeks in vitro, with the precise timing depending on culture conditions (Chen et al., 1996; Khirug et al., 2005). In our experiments, the glycine reversal potential after 14 $\mathrm{d}$ of culture was already hyperpolarizing and, more importantly, was the same as that in cultures in which GlyRs were chronically blocked with strychnine (Fig. 1D). Thus, our culture model system mimicked the first activation of newly expressed GlyRs on the cytoplasmic membrane after maturation of $\mathrm{Cl}^{-}$homeostasis when glycinergic transmission becomes dominant from GABAergic transmission in physiological conditions (Kotak et al., 1998; Gao et al., 2001; Russier et al., 2002). Indeed, the present results demonstrate that the activation-induced decrease of synaptic GlyR mobility that was associated with activation-dependent GlyR aggregation at synapses was not affected by $\mathrm{Ca}^{2+}$ chelation and inhibition of CaMKII (Fig. 5D,E). Although the activity-dependent cluster stabilization at the mature stage that we demonstrated here shares similarities with previous reports during early development, the underlying mechanisms must be distinct due to the lack of depolarization. Accordingly, our results propose that presynaptic release of inhibitory neurotransmitters alone is sufficient to regulate postsynaptic localization of inhibitory neurotransmitter receptors. Furthermore, this $\mathrm{Ca}^{2+}$-independent clustering of inhibitory neurotransmitter receptors might contribute to the homeostatic turnover of the postsynaptic receptors in mature synapses.

Neural activity and NMDA receptor activation decreases the lateral diffusion of GlyRs and increases their accumulation into synaptic clusters (Kirsch and Betz, 1998; Lévi et al., 2008). A remarkable observation in the present study is the speed at which this can occur. Only $1 \mathrm{~h}$ of GlyR activation was sufficient to restrict GlyR diffusion to synapses and to stabilize and increase functional clusters. Our experiments were all conducted in the continued presence of TTX, DL-APV, CNQX, and SR95531, excluding any more general changes in neural activity and excitatory receptor activation as contributors to this receptor stabilization. In addition, the interevent intervals of mIPSCs were not affected by chronic strychnine administration, indicating no prolonged changes in the neural circuit excitability (Fig. 1H). Hence, GlyR activation by itself stabilizes synaptic GlyRs, independent of changes in overall levels of excitatory neural activity.

GlyR diffusion properties and synaptic localization is known to depend critically on interactions with gephyrin, the scaffolding protein of inhibitory neurotransmitter receptors (Tyagarajan and Fritschy, 2014). In the present study, direct measurement of endogenous GlyR movements by single-particle tracking demonstrated that chronic receptor inactivation resulted in larger diffusion coefficients and an increase in the area over which GlyRs moved (Fig. 6B,C,E). When GlyR activation was allowed, the diffusive property of those GlyRs at synaptic domains decreased (Figs. 6, 7). The restricted mobility of activated GlyRs at synapses is likely to also involve interactions with gephyrin, as the mobility of GlyRs outside gephyrin clusters (in control condition) was much higher and was similar to that seen with chronic blockade of GlyRs (Fig. $4 D-G)$. Gephyrin interaction with the GlyR $\beta$ subunit has been suggested to decrease the lateral mobility of GlyRs (Schrader et al., 2004; Specht et al., 2011) through a phosphorylation-dependent mechanism (Zita et al., 2007; Charrier et al., 2010). And PKC-mediated phosphorylation enhanced postsynaptic localization of $\mathrm{GABA}_{A}$ receptors (Bannai et al., 2015). In the present study, treatment of the PKC inhibitor, but not the $\mathrm{Ca}^{2+}$ chelator, disturbed activation-dependent GlyR stabilization at postsynapses (Fig. $5 D, E$ ), suggesting that activation-dependent postsynaptic GlyR clustering in mature neurons may be mediated by the activity of $\mathrm{Ca}^{2+}$-independent PKC isoforms. Indeed, enhancement of glycinergic responses via PKC activation in hippocampal neurons (Schönrock and Bormann, 1995), and more specifically by $\mathrm{Ca}^{2+}$-independent PKC activity in sacral dorsal commissural nucleus, has been reported (Xu et al., 1996). In addition, ligand-induced conformational changes in GlyRs resulting from their activation may also mediate interactions with gephyrin (or associated proteins) and account for increased stability and cluster size (Meier, 2003). A structural analysis of 
GlyR block over 14 days
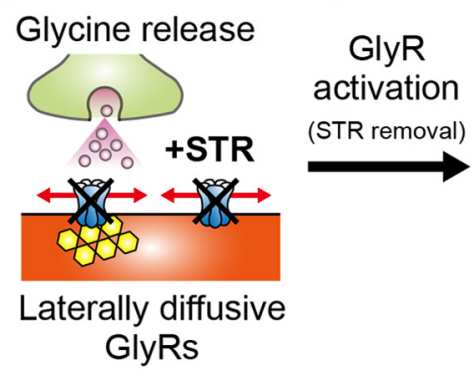

GlyRs

\section{GlyR activation (1h)}

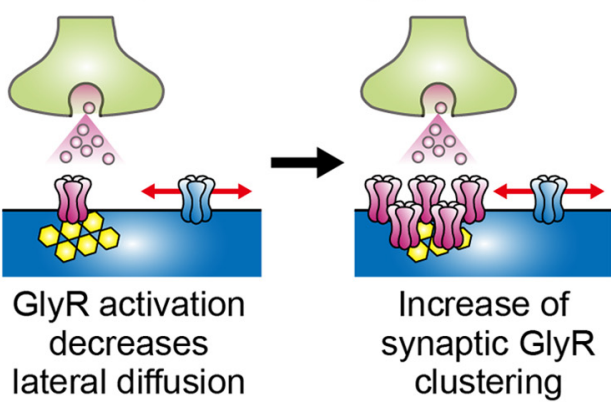

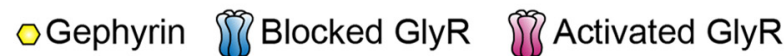

Figure 7. Summary diagram of activation-dependent postsynaptic GlyR clustering. (Left) Antagonized GlyRs by chronic application of strychnine (STR) are highly diffusive even at synaptic site in the presence of gephyrin, a scaffolding protein. (Middle) Strychnine removal allows GlyRs to be activated and decreases lateral diffusion at synaptic sites. (Right) Density of GlyRs increases at synaptic sites due to relatively lower diffusive properties than the extrasynaptic area.

GlyR using electron cryomicroscopy (Du et al., 2015) has recently reported that ligand binding induces dynamic changes in overall architecture, and the activationinduced conformational changes extend across both $\alpha$ and $\beta$-subunits (Lynch, 2004). Indeed, such activationdependent conformational changes affecting receptor clustering have been reported for the epidermal growth factor receptor (Ichinose et al., 2004), and a similar mechanism in ligand-gated neurotransmitter receptors was also recently reported for both AMPA receptors (Constals et al., 2015) and $\mathrm{GABA}_{\mathrm{A}}$ receptors (Gouzer et al., 2014; Lévi et al., 2015). Therefore, activation-induced conformational changes might result in the synaptic clustering of GlyRs in mature neurons.

Although GlyR activation rapidly enhances the synaptic localization of the receptors, interestingly, strychnineinduced GlyR inactivation needs a longer time to increase mobile fractions of previously activated GlyRs at mature synapses (Fig. 5B,C). While the present observation seems inconsistent with the previous report demonstrating that strychnine application for $24 \mathrm{~h}$ decreases the density of previously clustered GlyRs in zebrafish Mauthner cells, this effect of strychnine on the GlyR density is reduced with animal development (Yamanaka et al., 2013). Indeed, Seitanidou et al. (1992) also reported that localizations of GlyRs are less affected by strychnine treatment in adult animals in vivo. These previous reports and our present study suggest that once glycine receptors are activated at postsynaptic sites, they can stay for longer durations in the mature stage. Although the stable localization of GlyR at synapse over $36 \mathrm{~h}$ seems longer than the half-life of surface GlyRs (Rasmussen et al., 2002), the previous study measured the turnover rate of nonspecific GlyR subtypes from all of the cell surface, including extrasynaptic GlyRs. Indeed, turnover rates of exocytosis/endocytosis in AMPA receptors and GABA $_{A}$ receptors are higher in the extrasynaptic area than at synapses (Bogdanov et al., 2006; Opazo and Choquet, 2011), and this is thought to occur in glycine receptors (Rosenberg et al., 2001; Dumoulin et al., 2009). Thus, the long-lasting stability of GlyRs observed in the present study may suggest that the localization of $\alpha 1$-subunitcontained GlyRs is robust at synapses. Furthermore, the increased mobile fraction of GlyRs, which were treated with strychnine for $48 \mathrm{~h}$, is comparable to the cSTR condition, indicating that the half-life of stabilized GlyRs at synapses is nearly $24 \mathrm{~h}$. Although activation-dependent postsynaptic GlyR stabilization occurs even within $1 \mathrm{~h}$, the destabilization of previously activated GlyR takes over 24 $\mathrm{h}$. This discrepancy in the time required for increasing or decreasing postsynaptic stabilization may suggest that GlyR activation is more important for the formation of synaptic clustering of GlyR rather than for maintenance in mature neurons.

In spinal cord and brainstem, the dynamic shift of released inhibitory neurotransmitter contents occurs during development in presynapses (Nabekura et al., 2004). In parallel, postsynaptic modulation is necessary for the fine-tuning of inhibitory synaptic efficacy in mature neurons. It is known that predominant subunit composition of GlyRs developmentally shifts from homomeric GlyR $\alpha 2$ to heteromeric GlyR $\alpha 1 \beta$ (Lynch, 2004). Hence, the $\beta$-subunit of GlyRs that bind to gephyrin enables localization to synaptic sites (Tyagarajan and Fritschy, 2014). In addition to the developmental changes in GlyR subunit composition, the present study demonstrates that GlyR activation is critical for the postsynaptic clustering of the receptor. Therefore, the formation of appropriate inhibitory synapse in mature neurons occurs in both presynapses and postsynapses. And postsynaptic receptor distributions are constantly regulated by released neurotransmitters in an activation-dependent manner without $\mathrm{Ca}^{2+}$-mediated depolarizing responses in the mature neural system.

Together, activation-dependent synaptic clustering of GlyRs in mature neurons might be mediated by modulating the interactions between GlyRs and gephyrin via PKCmediated signaling. Although the precise mechanisms of activation-dependent GlyR dynamics and gephyrin interactions remain to be fully elucidated, our data demonstrate that activation-dependent postsynaptic GlyR clustering rapidly occurs in mature neurons and may be involved with mechanisms different from those at developing synapses. 
The dynamic molecular changes we report here are likely to contribute to a postsynaptic mechanism underlying the developmental switching of inhibitory neurotransmission and the activity-dependent functional strengthening of inhibitory synaptic transmission in the adult CNS.

\section{References}

Ashby MC, Ibaraki K, Henley JM (2004) It's green outside: tracking cell surface proteins with $\mathrm{pH}$-sensitive GFP. Trends Neurosci 27: 257-261. CrossRef Medline

Bannai H, Lévi S, Schweizer C, Dahan M, Triller A (2006) Imaging the lateral diffusion of membrane molecules with quantum dots. Nat Protoc 1:2628-2634. CrossRef Medline

Bannai H, Lévi S, Schweizer C, Inoue T, Launey T, Racine V, Sibarita JB, Mikoshiba K, Triller A (2009) Activity-dependent tuning of inhibitory neurotransmission based on $\mathrm{GABA}_{A} \mathrm{R}$ diffusion dynamics. Neuron 62:670-682. CrossRef Medline

Bannai H, Niwa F, Sherwood MW, Shrivastava AN, Arizono M, Miyamoto A, Sugiura K, Lévi S, Triller A, Mikoshiba K (2015) Bidirectional control of synaptic GABAAR clustering by glutamate and calcium. Cell Rep 13:2768-2780. CrossRef Medline

Becker CM, Schmieden V, Tarroni P, Strasser U, Betz H (1992) Isoform-selective deficit of glycine receptors in the mouse mutant spastic. Neuron 8:283-289. Medline

Ben-Ari Y (2002) Excitatory actions of GABA during development: the nature of the nurture. Nat Rev Neurosci 3:728-739. CrossRef Medline

Bogdanov Y, Michels G, Armstrong-Gold C, Haydon PG, Lindstrom J, Pangalos M, Moss SJ (2006) Synaptic GABAA receptors are directly recruited from their extrasynaptic counterparts. EMBO $\mathrm{J}$ 25:4381-4389. CrossRef Medline

Bonneau S, Dahan M, Cohen LD (2005) Single quantum dot tracking based on perceptual grouping using minimal paths in a spatiotemporal volume. IEEE Trans Image Process 14:1384-1395. CrossRef

Borgdorff AJ, Choquet D (2002) Regulation of AMPA receptor lateral movements. Nature 417:649-653. CrossRef Medline

Calamai M, Specht CG, Heller J, Alcor D, Machado P, Vannier C, Triller A (2009) Gephyrin oligomerization controls GlyR mobility and synaptic clustering. J Neurosci 29:7639-7648. CrossRef Medline

Charrier C, Machado P, Tweedie-Cullen RY, Rutishauser D, Mansuy IM, Triller A (2010) A crosstalk between $\beta 1$ and $\beta 3$ integrins controls glycine receptor and gephyrin trafficking at synapses. Nat Neurosci 13:1388-1395. CrossRef Medline

Chen G, Trombley PQ, van den Pol AN (1996) Excitatory actions of GABA in developing rat hypothalamic neurones. J Physiol 494: 451-464. CrossRef

Choquet D, Triller A (2013) The dynamic synapse. Neuron 80:691703. CrossRef Medline

Constals A, Penn AC, Compans B, Toulmé E, Phillipat A, Marais S, Retailleau N, Hafner AS, Coussen F, Hosy E, Choquet D (2015) Glutamate-induced AMPA receptor desensitization increases their mobility and modulates short-term plasticity through unbinding from Stargazin. Neuron 85:787-803. CrossRef Medline

Dahan M, Lévi S, Luccardini C, Rostaing P, Riveau B, Triller A (2003) Diffusion dynamics of glycine receptors revealed by singlequantum dot tracking. Science 302:442-445. CrossRef Medline

Du J, Lü W, Wu S, Cheng Y, Gouaux E (2015) Glycine receptor mechanism elucidated by electron cryo-microscopy. Nature 526: 224-229. CrossRef Medline

Dumoulin A, Triller A, Kneussel M (2009) Cellular transport and membrane dynamics of the glycine receptor. Front Mol Neurosci 2:28. CrossRef Medline

Ebihara S, Shirato K, Harata N, Akaike N (1995) Gramicidinperforated patch recording: GABA response in mammalian neurones with intact intracellular chloride. J Physiol 484:77-86. CrossRef
Ehrlich I, Lohrke S, Friauf E (1999) Shift from depolarizing to hyperpolarizing glycine action in rat auditory neurones is due to agedependent Cl- regulation. J Physiol 520:121-137. CrossRef

Friauf E, Hammerschmidt B, Kirsch J (1997) Development of adulttype inhibitory glycine receptors in the central auditory system of rats. J Comp Neurol 385:117-134. Medline

Fujiwara T, Oda K, Yokota S, Takatsuki A, Ikehara Y (1988) Brefeldin A causes disassembly of the Golgi complex and accumulation of secretory proteins in the endoplasmic reticulum. J Biol Chem 263:18545-18552.

Gaffield MA, Betz WJ (2006) Imaging synaptic vesicle exocytosis and endocytosis with FM dyes. Nat Protoc 1:2916-2921. CrossRef Medline

Gao BX, Stricker C, Ziskind-Conhaim L (2001) Transition from GABAergic to glycinergic synaptic transmission in newly formed spinal networks. J Neurophysiol 86:492-502. Medline

Gouzer G, Specht CG, Allain L, Shinoe T, Triller A (2014) Benzodiazepine-dependent stabilization of GABA(A) receptors at synapses. Mol Cell Neurosci 63:101-113. CrossRef Medline

Hildick KL, González-González IM, Jaskolski F, Henley JM (2012) Lateral diffusion and exocytosis of membrane proteins in cultured neurons assessed using fluorescence recovery and fluorescenceloss photobleaching. J Vis Exp 60:1-9.

Huganir RL, Nicoll RA (2013) AMPARs and synaptic plasticity: the last 25 years. Neuron 80:704-717. CrossRef Medline

Ichinose J, Murata M, Yanagida T, Sako Y (2004) EGF signalling amplification induced by dynamic clustering of EGFR. Biochem Biophys Res Commun 324:1143-1149. CrossRef Medline

Ishibashi H, Yamaguchi J, Nakahata Y, Nabekura J (2013) Dynamic regulation of glycine-GABA co-transmission at spinal inhibitory synapses by neuronal glutamate transporter. J Physiol 591:38213832. CrossRef Medline

Jacob TC, Moss SJ, Jurd R (2008) GABA(A) receptor trafficking and its role in the dynamic modulation of neuronal inhibition. Nat Rev Neurosci 9:331-343. CrossRef Medline

Jaskolski F, Mayo-Martin B, Jane D, Henley JM (2009) Dynamindependent membrane drift recruits AMPA receptors to dendritic spines. J Biol Chem 284:12491-12503. CrossRef Medline

Jean-Xavier C, Pflieger JF, Liabeuf S, Vinay L (2006) Inhibitory postsynaptic potentials in lumbar motoneurons remain depolarizing after neonatal spinal cord transection in the rat. $\mathrm{J}$ Neurophysiol 96:2274-2281. CrossRef

Kakazu Y, Akaike N, Komiyama S, Nabekura J (1999) Regulation of intracellular chloride by cotransporters in developing lateral superior olive neurons. J Neurosci 19:2843-2851.

Khirug S, Huttu K, Ludwig A, Smirnov S, Voipio J, Rivera C, Kaila K, Khiroug L (2005) Distinct properties of functional KCC2 expression in immature mouse hippocampal neurons in culture and in acute slices. Eur J Neurosci 21:899-904. CrossRef Medline

Kirsch J, Betz H (1995) The postsynaptic localization of the glycine receptor-associated protein gephyrin is regulated by the cytoskeleton. J Neurosci 15:4148-4156. Medline

Kirsch J, Betz H (1998) Glycine-receptor activation is required for receptor clustering in spinal neurons. Nature 392:717-720. CrossRef Medline

Kondratskaya EL, Betz H, Krishtal OA, Laube B (2005) The beta subunit increases the ginkgolide $B$ sensitivity of inhibitory glycine receptors. Neuropharmacology 49:945-951. CrossRef Medline

Korada S, Schwartz IR (1999) Development of GABA, glycine, and their receptors in the auditory brainstem of gerbil: a light and electron microscopic study. J Comp Neurol 409:664-681. Medline

Kotak VC, Korada S, Schwartz IR, Sanes DH (1998) A developmental shift from GABAergic to glycinergic transmission in the central auditory system. J Neurosci 18:4646-4655. Medline

Kuriu T, Yanagawa Y, Konishi S (2012) Activity-dependent coordinated mobility of hippocampal inhibitory synapses visualized with presynaptic and postsynaptic tagged-molecular markers. Mol Cell Neurosci 49:184-195. CrossRef Medline

Kusumi A, Sako Y, Yamamoto M (1993) Confined lateral diffusion of membrane receptors as studied by single particle tracking (nano- 
vid microscopy). Effects of calcium-induced differentiation in cultured epithelial cells. Biophys J 65:2021-2040. CrossRef Medline

Lévi S, Vannier C, Triller A (1998) Strychnine-sensitive stabilization of postsynaptic glycine receptor clusters. J Cell Sci 111:335-345.

Lévi S, Schweizer C, Bannai H, Pascual O, Charrier C, Triller A (2008) Homeostatic regulation of synaptic GlyR numbers driven by lateral diffusion. Neuron 59:261-273. CrossRef Medline

Lévi S, Le Roux N, Eugène E, Poncer JC (2015) Benzodiazepine ligands rapidly influence $\mathrm{GABA}_{\mathrm{A}}$ receptor diffusion and clustering at hippocampal inhibitory synapses. Neuropharmacology 88:199208. CrossRef Medline

Lynch JW (2004) Molecular structure and function of the glycine receptor chloride channel. Physiol Rev 84:1051-1095. CrossRef Medline

Lynch JW, Callister RJ (2006) Glycine receptors: a new therapeutic target in pain pathways. Curr Opin Investig Drugs 7:48-53. Medline

Ma W, Behar T, Chang L, Barker JL (1994) Transient increase in expression of GAD65 and GAD67 mRNAs during postnatal development of rat spinal cord. J Comp Neurol 346:151-160. CrossRef Medline

Manders EMM, Verbeek FJ, Aten JA (1993) Measurement of colocalization of objects in dual-colour confocal images. J Microsc 169:375-382. CrossRef

Matsubayashi H, Alkondon M, Pereira EF, Swanson KL, Albuquerque EX (1998) Strychnine: a potent competitive antagonist of alphabungarotoxin-sensitive nicotinic acetylcholine receptors in rat hippocampal neurons. J Pharmacol Exp Ther 284:904-913. Medline

McIntire SL, Reimer RJ, Schuske K, Edwards RH, Jorgensen EM (1997) Identification and characterization of the vesicular GABA transporter. Nature 389:870-876. CrossRef Medline

Meier J (2003) The enigma of transmitter-selective receptor accumulation at developing inhibitory synapses. Cell Tissue Res 311: 271-276. CrossRef Medline

Miesenböck G, De Angelis DA, Rothman JE (1998) Visualizing secretion and synaptic transmission with $\mathrm{pH}$-sensitive green fluorescent proteins. Nature 394:192-195. CrossRef Medline

Murase K, Ryu PD, Randic M (1989) Excitatory and inhibitory amino acids and peptide-induced responses in acutely isolated rat spinal dorsal horn neurons. Neurosci Lett 103:56-63. Medline

Nabekura J, Katsurabayashi S, Kakazu Y, Shibata S, Matsubara A, Jinno S, Mizoguchi Y, Sasaki A, Ishibashi H (2004) Developmental switch from GABA to glycine release in single central synaptic terminals. Nat Neurosci 7:17-23. CrossRef Medline

Opazo P, Choquet D (2011) A three-step model for the synaptic recruitment of AMPA receptors. Mol Cell Neurosci 46:1-8. CrossRef Medline

Papke RL, Sanberg PR, Shytle RD (2001) Analysis of mecamylamine stereoisomers on human nicotinic receptor subtypes. J Pharmacol Exp Ther 297:646-656. Medline

Petrini EM, Ravasenga T, Hausrat TJ, lurilli G, Olcese U, Racine V, Sibarita JB, Jacob TC, Moss SJ, Benfenati F, Medini P, Kneussel $M$, Barberis A (2014) Synaptic recruitment of gephyrin regulates surface $\mathrm{GABA}_{A}$ receptor dynamics for the expression of inhibitory LTP. Nat Commun 5:3921. CrossRef

Rannals MD, Kapur J (2011) Homeostatic strengthening of inhibitory synapses is mediated by the accumulation of $\mathrm{GABA}_{\mathrm{A}}$ receptors. $\mathrm{J}$ Neurosci 31:17701-17712. CrossRef Medline

Rasmussen H, Rasmussen T, Triller A, Vannier C (2002) Strychnineblocked glycine receptor is removed from synapses by a shift in insertion/degradation equilibrium. Mol Cell Neurosci 19:201-215. CrossRef Medline

Reits EA, Neefjes JJ (2001) From fixed to FRAP: measuring protein mobility and activity in living cells. Nat Cell Biol 3:E145-E147. CrossRef Medline

Rivera C, Voipio J, Payne JA, Ruusuvuori E, Lahtinen H, Lamsa K, Pirvola U, Saarma M, Kaila K (1999) The K+/Cl- co-transporter
KCC2 renders GABA hyperpolarizing during neuronal maturation. Nature 397:251-255. CrossRef

Rosenberg M, Meier J, Triller A, Vannier C (2001) Dynamics of glycine receptor insertion in the neuronal plasma membrane. $\mathrm{J}$ Neurosci 21:5036-5044. Medline

Russier M, Kopysova IL, Ankri N, Ferrand N, Debanne D (2002) GABA and glycine co-release optimizes functional inhibition in rat brainstem motoneurons in vitro. J Physiol 541:123-137. Medline

Sanes DH, Malone BJ, Semple MN (1998) Role of synaptic inhibition in processing of dynamic binaural level stimuli. J Neurosci 18:794803. Medline

Sankaranarayanan S, Ryan TA (2001) Calcium accelerates endocytosis of vSNAREs at hippocampal synapses. Nat Neurosci 4:129136. CrossRef Medline

Saxton MJ, Jacobson K (1997) Single-particle tracking: applications to membrane dynamics. Annu Rev Biophys Biomol Struct 26:373399. CrossRef Medline

Schönrock B, Bormann J (1995) Modulation of hippocampal glycine receptor channels by protein kinase C. Neuroreport 6:301-304. Medline

Schrader N, Kim EY, Winking J, Paulukat J, Schindelin H, Schwarz G (2004) Biochemical characterization of the high affinity binding between the glycine receptor and gephyrin. J Biol Chem 279: 18733-18741. CrossRef Medline

Seitanidou T, Nicola MA, Triller A, Korn H (1992) Partial glycinergic denervation induces transient changes in the distribution of a glycine receptor-associated protein in a central neuron. J Neurosci 12:116-131.

Soumpasis DM (1983) Theoretical analysis of fluorescence photobleaching recovery experiments. Biophys J 41:95-97. CrossRef Medline

Specht CG, Grünewald N, Pascual O, Rostgaard N, Schwarz G, Triller A (2011) Regulation of glycine receptor diffusion properties and gephyrin interactions by protein kinase C. EMBO J 30:38423853. CrossRef Medline

Tyagarajan SK, Fritschy JM (2014) Gephyrin: a master regulator of neuronal function? Nat Rev Neurosci 15:141-156. CrossRef Medline

Waataja JJ, Kim HJ, Roloff AM, Thayer SA (2008) Excitotoxic loss of post-synaptic sites is distinct temporally and mechanistically from neuronal death. J Neurochem 104:364-375. CrossRef Medline

Wang Y, Kakizaki T, Sakagami H, Saito K, Ebihara S, Kato M, Hirabayashi M, Saito Y, Furuya N, Yanagawa Y (2009) Fluorescent labeling of both GABAergic and glycinergic neurons in vesicular GABA transporter (VGAT)-venus transgenic mouse. Neuroscience 164:1031-1043. CrossRef Medline

Weltzien F, Puller C, O'Sullivan GA, Paarmann I, Betz H (2012) Distribution of the glycine receptor $\beta$-subunit in the mouse CNS as revealed by a novel monoclonal antibody. J Comp Neurol 520: 3962-3981. CrossRef Medline

Xu TL, Nabekura J, Akaike N (1996) Protein kinase C-mediated enhancement of glycine response in rat sacral dorsal commissural neurones by serotonin. J Physiol 496:491-501. CrossRef

Yamanaka I, Miki M, Asakawa K, Kawakami K, Oda Y, Hirata H (2013) Glycinergic transmission and postsynaptic activation of CaMKII are required for glycine receptor clustering in vivo. Genes Cells 18:211-224. CrossRef Medline

Yasuyoshi H, Kashii S, Kikuchi M, Zhang S, Honda Y, Akaike A (2002) New insight into the functional role of acetylcholine in developing embryonic rat retinal neurons. Invest Ophthalmol Vis Sci 43:446451. Medline

Zita MM, Marchionni I, Bottos E, Righi M, Del Sal G, Cherubini E, Zacchi P (2007) Post-phosphorylation prolyl isomerisation of gephyrin represents a mechanism to modulate glycine receptors function. EMBO J 26:1761-1771. CrossRef Medline 